\title{
Efficient Simulation of Lévy-driven Point Processes*
}

\author{
Angelos Dassios ${ }^{\dagger}$ \\ Yan $\mathrm{Qu}^{*}$ \\ London School of Economics \\ London School of Economics \\ Hongbiao Zhao ${ }^{\S}$ \\ Shanghai University of Finance and Economics
}

\begin{abstract}
In this paper, we introduce a new large family of Lévy-driven point processes with (and without) contagion, by generalising the classical self-exciting Hawkes process and doubly stochastic Poisson processes with non-Gaussian Lévy-driven Ornstein-Uhlenbeck type intensities. The resulting framework may possess many desirable features such as skewness, leptokurtosis, mean-reverting dynamics, and more importantly, the "contagion" or feedback effects, which could be very useful for modelling event arrivals in finance, economics, insurance and many other fields. We characterise the distributional properties of this new class of point processes and develop an efficient sampling method for generating sample paths exactly. Our simulation scheme is mainly based on the distributional decomposition of the point process and its intensity process. Extensive numerical implementations and tests are reported to demonstrate the accuracy and effectiveness of our scheme. Moreover, we apply to portfolio risk management as an example to show the applicability and flexibility of our algorithms.
\end{abstract}

Keywords: Contagion risk; Portfolio risk management; Monte Carlo simulation; Exact simulation; Exact decomposition; Self-exciting jump process with non-Gaussian Ornstein-Uhlenbeck intensity; Point process; Branching process; Stochastic intensity model; Non-Gaussian Ornstein-Uhlenbeck process; Gamma process; Inverse Gaussian subordinator; Tempered stable subordinator

Mathematics Subject Classification (2010): Primary: 60G55 - 62E15 - 65C05; Secondary: 60E07 · $60 \mathrm{G} 51$ $\cdot 60 \mathrm{~J} 75 \cdot 60 \mathrm{H} 35$

\footnotetext{
*An edited version to be published by Applied Probability Journals

† Department of Statistics, London School of Economics, Houghton Street, London WC2A 2AE, United Kingdom. Email: a.dassios@1se.ac.uk

Department of Statistics, London School of Economics, Houghton Street, London WC2A 2AE, United Kingdom. Email:y.qu3@1se.ac.uk

${ }^{\S}$ Corresponding author, School of Statistics and Management, Shanghai University of Finance and Economics, No. 777 Guoding Road, Shanghai 200433, China; Shanghai Institute of International Finance and Economics, No. 777 Guoding Road, Shanghai 200433, China. Email: h.zhao1@1se.ac.uk
} 


\section{Introduction}

Doubly stochastic Poisson processes or Cox processes (Cox, 1955, 1972) have now been widely applied as survival or event timing models in many areas. They are more capable than a simple Poisson process to capture event arrivals with complex dynamics structures. However, in reality, except for the impact from external factors, event arrivals may often present "contagion", clustering, or feedback effects, such as social media sharing online, trade transactions in market microstructure, defaults in the credit market, jumps in investment returns, and loss claims in insurance businesses to name a few. Das et al. (2007) and Duffie et al. (2009) provided evidences that, Cox models, which are based on conditional independence assumption, can not fully capture credit contagion. The phenomena of contagion became more evident in the credit market during the global financial crisis of 2007-09 and European debt crisis since the end of 2009 (Giesecke et al., 2011b). A seminal framework tailored for modelling event contagion is Hawkes process (Hawkes, 1971a,b). It is a self-exciting point process where each arrival of events would trigger a simultaneous jump in its own intensity and hence more events follow. Empirical evidence and econometric analysis can be viewed from Bowsher (2007), Large (2007), Crane and Sornette (2008), Errais et al. (2010), Embrechts et al. (2011), Bacry et al. (2013), Aït-Sahalia et al. (2014, 2015) and Azizpour et al. (2018). Recently, it has been extended in literature by being combined with Cox processes to enrich the model eligibility, in the sense that both internal and external impacts can be facilitated in one single framework, see Brémaud and Massoulié (1996, 2002) and Dassios and Zhao (2011, 2017b).

Meanwhile, from a micro perspective, it becomes more apparent that real financial data exhibits deviations from normality with the availability of high-frequency data ${ }^{1}$. Barndorff-Nielsen and Shephard (2001a,b) proposed a class of stochastic processes, namely non-Gaussian OrnsteinUhlenbeck $(\mathrm{OU})$ models, which have gained extensive popularity for modelling the non-normality presented in finance and economics. They could offer greater flexibility and possess many crucial features, such as skewness, leptokurtosis and mean-reverting dynamics, which are often observed from financial markets ${ }^{2}$. Moreover, this generality does not hinder their analytical tractability. In particular, they become extremely popular for modelling stochastic volatilities, see BarndorffNielsen et al. (1998), Barndorff-Nielsen and Shephard (2001a,b, 2002, 2003a,b) and Carr et al. (2003). These stochastic volatility models have further led to other applications such as derivative pricing and risk analysis, see Nicolato and Venardos (2003) and Li and Linetsky (2014). On the other hand, these processes can serve as stochastic intensity models for event arrivals. For in-

\footnotetext{
${ }^{1}$ See a pioneering investigation into the high-frequency financial data by Gençay et al. (2001) and recent advances in financial econometrics in Ait-Sahalia and Jacod (2014).

${ }^{2}$ See empirical evidences from Poterba and Summers (1988) and Cont (2001).
} 
stance, they have been used to model irregularly-spaced trade-by-trade intraday data, mortality rates in insurance, and default intensities for credit risk in finance, see Rydberg and Shephard (2000), Centanni and Minozzo (2006), Hainaut and Devolder (2008) and Schoutens and Cariboni (2010). In particular, for credit risk modelling, a mean-reverting OU intensity could be particularly useful to capture business cycle effects on average industrial defaults, as obviously default rates would increase in a recession and decrease in a boom (Elsinger et al., 2006, p.1306). This is similar as the environment of interest rates, so, defaults and the associated losses in the credit market often present a mean-reverting pattern, see detailed analysis and evidence in Giesecke et al. (2011b) from a long-term historical perspective. Duffie et al. (2009) also found a mean-reverting frailty that would influence U.S. non-financial defaults. Moreover, empirical evidence shows that, the tails of Gaussian distributions are often too thin to capture risk in the credit market, and fluctuations are often sudden and jump-like, which are driven by unexpected news announcements. The distribution of default rates is highly skewed towards large values (Giesecke et al., 2011b, p.236-239). Therefore, macroeconomic shocks powered by a Lévy-driven non-Gaussian process rather than a Gaussian one may be more appropriate to capture the dynamic structure of default intensities in reality.

It is then natural for us to put these main streamlines above in the literature together to form a unified and consistent framework. In this paper, we construct a new large family of Lévy-driven point processes, termed self-exciting jump process with non-Gaussian OU intensity, or, Lévy-driven contagion process. It is fundamentally powered by a Lévy process. More precisely, its stochastic intensity is a positive non-Gaussian process with additional self-exciting jumps. It can be also defined as a branching process through the cluster process representation. Accordingly, the resulting models are analytically tractable, and intrinsically inherit the great flexibility as well as the desirable features from the two original processes, including skewness, leptokurtosis, mean-reverting dynamics, and more importantly, the "contagion" or feedback effects. These newly-constructed processes would then substantially enrich continuous-time models tailored for quantifying the "contagion" of event arrivals in finance, economics, insurance, queueing and many other fields.

Simulation plays a crucial role in the implementation, simulation-based statistical inference and empirical studies for new models. For instance, for modelling credit risk in practice, events of extreme losses and defaults are rare, and data are scarce. The key quantities at the center of financial risk management, such as the value at risk of an aggregate loss distribution for a heterogenous portfolio, are often lack of closed-form formulas. Simulation-based approach then becomes a standard technique. In particular, the exact simulation scheme is highly desirable as it has the primary ad- 
vantage of generating sample paths according to the underlying process law exactly (Broadie and Kaya, 2006; Chen and Huang, 2013). To our knowledge, there is no exact simulation algorithms in the literature even for our important special cases, the non-Gaussian OU stochastic intensity models without contagion. We first propose a general sampling framework for exactly simulating Lévy-driven contagion processes based on decomposition technique ${ }^{3}$. Then, we focus on exploiting some very typical specifications of Lévy processes such as gamma process and tempered stable subordinator, where remarkably the resulting point processes can be analytically decomposed into several types of basic components, each of which can be exactly simulated, either directly, or via an acceptance-rejection $(A / R)$ scheme. Even though the underlying process possess a complex structure, neither procedure of truncation nor discretisation is required. Moreover, there is no numerical inversion for the cumulative distribution function (CDF) or Laplace (Fourier) transform.

The paper is organised as follows. Section 2 gives the mathematical definitions of this new family, and explains how they can be constructed from the classical non-Gaussian OU processes and the self-exciting point process. In Section 3, we develop a general framework for exactly sampling our new point processes. In Section 4, we derive the exact simulation algorithms based on decomposition approach for two explicit cases when the driving Lévy processes are gamma process and tempered stable subordinator, respectively. Extensive numerical implementations and tests are reported in Section 5 to demonstrate the accuracy and effectiveness of our algorithms. Section 6 provides some financial applications to credit portfolio risk to show the applicability and flexibility of our algorithms. Finally, Section 7 draws a brief conclusion and suggests plenty potential topics for future research based on this new framework.

\section{Lévy-driven Contagion Models}

In this section, we construct a new framework for modelling event arrivals with contagion effects based on Lévy processes. That is, the intensity of the point process is set up to be a non-Gaussian OU process driven by a Lévy subordinator in cooperation with extra self-exciting jumps. Let us first define a simpler version without the self-exciting component:

Definition 2.1 (Jump Process with Non-Gaussian Intensity). Jump process with non-Gaussian intensity is a point process $N \equiv\left\{T_{i}\right\}_{i=1,2, \ldots}$ i.e. $N_{t}=\sum_{i \geq 1} \mathbf{1}_{\left\{T_{i} \leq t\right\}}$ with the stochastic intensity $\lambda_{t}$

\footnotetext{
${ }^{3}$ This decomposition approach has also been recently used by Dassios and Zhao (2013, 2017a) to simulate the classical Hawkes process and point process with CIR intensity and Dassios et al. (2018); ?); ? to simulate tempered stable distributions and gamma-driven Ornstein-Uhlenbeck processes.
} 
satisfying the stochastic differential equation

$$
\mathrm{d} \lambda_{t}=-\delta \lambda_{t} \mathrm{~d} t+\varrho \mathrm{d} Z_{t}, \quad t \geq 0
$$

where

- $\varrho>0$ is an arbitrary positive constant;

- $\delta>0$ is the constant rate of exponential decay;

- $Z_{t}$, with $Z_{0}=0$, is a Lévy subordinator, which is called the background driving Lévy process (BDLP) of a non-Gaussian OU process.

This is a special case of Cox point processes. A slightly mathematical generalisation but very useful for applications is to further incorporate a feedback mechanism in the framework by adding a series of self-exciting jumps, i.e. simultaneous jumps (or "co-jumps") in the point process and its intensity process. More precisely, this new framework, as a generalised version of the jump process of Definition 2.1, is defined via the stochastic intensity representation as below:

Definition 2.2 (Self-exciting Jump Process with Non-Gaussian Intensity). $N_{t}$ is a self-exciting jump process with non-Gaussian intensity, if the intensity process of (2.1) is replaced by

$$
\mathrm{d} \lambda_{t}=-\delta \lambda_{t} \mathrm{~d} t+\varrho \mathrm{d} Z_{t}+\mathrm{d} J_{t}, \quad t \geq 0,
$$

where the extra component $J_{t}$ is a pure-jump process specified by

$$
J_{t}:=\sum_{i=1}^{N_{t}} X_{i}
$$

and $\left\{X_{i}\right\}_{i=1,2, \ldots}$ are the sizes of self-exciting jumps ${ }^{4}$ with $\mathcal{F}_{T_{i}^{-}}$-measurable ${ }^{5}$ distribution function $G(z), z>0$, occurring at the associated (ordered) arrival times $\left\{T_{i}\right\}_{i=1,2, \ldots}$, respectively, and they are independent of $Z_{t}$.

Similar as the Hawkes process (Hawkes and Oakes, 1974), $N_{t}$ in Definition 2.2 can be equivalently redefined as a branching process via a cluster process presentation (Daley and Vere-Jones,

\footnotetext{
${ }^{4}$ It is called "self-exciting", as the expression (2.3) reveals that the jumps simultaneously occur in the point process $N_{t}$ and its intensity $\lambda_{t}$, and hence the arrivals of jumps trigger more jumps afterwards.

${ }^{5}$ It means that the functional form of the distribution function $G(z)$ is revealed just before the arrival time $T_{i}$. This distribution could have a highly general dependency structure $G(z)=G(z \mid \cdot)$. For example, it could depend on the initial intensity $\lambda_{0}$, the past history of intensity at or just before the jump arrival times $\left\{T_{k}\right\}_{k=1,2, \ldots, i}$, all past jump sizes $\left\{X_{k}\right\}_{k=1,2, \ldots, i-1}$, or the cumulated number of jumps $N_{t}$, and so on, as long as we can record these information, for example,

$$
G(z)=G\left(z \mid T_{1}, T_{2}, \ldots, T_{i}, \lambda_{0}, \lambda_{T_{1}^{-}}, \ldots, \lambda_{T_{i}^{-}}, \lambda_{T_{1}}, \ldots, \lambda_{T_{i-1}}\right) .
$$

This is similar as the adaptive model setting of Giesecke et al. (2011a), but it is different from the classical Hawkes process.
} 
2003, p.175-193). More precisely, $N_{t}$ is a cluster point process which consists of two types of points: immigrants and their offspring. The arrivals of immigrants follow a Cox process with nonGaussian OU intensity (2.1). Each immigrant generates its offspring, each offspring would further generate offspring, and so on. The generation of any offspring follows a Cox process with exponentially decaying intensity $X^{*} e^{-\delta\left(t-T^{*}\right)}$, where $X^{*} \stackrel{\mathcal{D}}{=} X_{i}$ and $T^{*}$ is the arrival (birth) time of its previous generation. The superposition (Daley and Vere-Jones, 2003, Theorem 2.4.VI) of all of these points forms our new self-exciting point process $N_{t}$ with the stochastic intensity (2.2).

Note that, given the initial intensity level $\lambda_{0}>0$, the intensity process (2.2) can be equivalently expressed as

$$
\lambda_{t}=\underbrace{\lambda_{0} e^{-\delta t}+\varrho \int_{0}^{t} e^{-\delta(t-s)} \mathrm{d} Z_{s}}_{\text {Exogenous commonly-shared risk }}+\underbrace{\sum_{i=1}^{N_{t}} e^{-\delta\left(t-T_{i}\right)} X_{i}}_{\text {Endogenous contagion risk }}, \quad t \geq 0
$$

which is positive and càdlàg. In fact, this new framework integrates two major types of risk sources. For example, in the context of credit risk or systemic risk, the first part (i.e. the first two terms) is to model the cyclical dependency of companies on some exogenous risk (e.g. movements of interest or FX rates) commonly shared in the entire market, and the cyclical oscillation is captured by the mean-reverting non-Gaussian OU process; fundamental common shocks are captured by the pure-jump process $Z_{t}$. The second part (i.e. the last term) is to model the endogenous contagion risk due to the local interaction of companies in their business network, without which the overall risk would be underestimated.

The interarrival intensity process $\left\{\lambda_{t}\right\}_{T_{i} \leq t<T_{i+1}}$, for modelling exogenous commonly-shared risk, is defined as the parts of intensity process excluding self-exciting jumps, i.e., (2.1), or,

$$
\lambda_{t}=e^{-\delta t} \lambda_{0}+\varrho \int_{0}^{t} e^{-\delta(t-s)} \mathrm{d} Z_{s}, \quad t \in\left[T_{i}, T_{i+1}\right)
$$

For instance, a sample path of the interarrival intensity process (without self-exciting component) within the time period $t \in[0,5]$ when the BDLP is a gamma process is represented in Figure 1.

This framework is the generalisation of several classical models in the literature: If there is no self-exciting jumps, i.e. $X_{i} \equiv 0$ for any $i$, then, the intensity process (2.2) is a non-Gaussian $O U$ process (Barndorff-Nielsen et al., 1998; Barndorff-Nielsen and Shephard, 2001a,b, 2002, 2003a). If there is no BDLP $Z_{t}$, then, the point process $N_{t}$ is a generalised Hawkes process (Hawkes, 


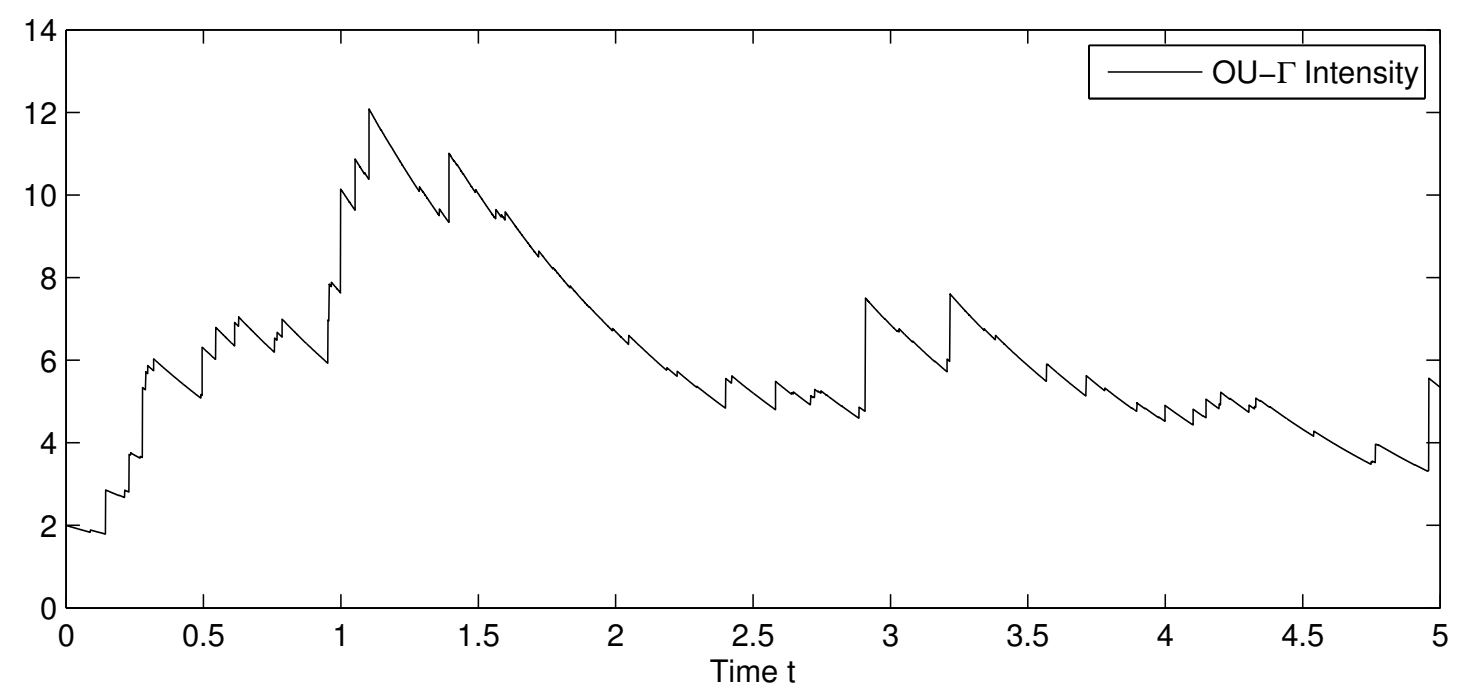

Figure 1: A sample path of interarrival intensity process (without self-exciting component) within the time period $t \in[0,5]$ when the BDLP is a gamma process

1971a,b) with random marks. If the BDLP $Z_{t}$ is trivially a subordinator of compound Poisson, then, $N_{t}$ is a dynamic contagion process (Dassios and Zhao, 2011).

For notation simplicity, we denote the Lévy measure of BDLP $Z_{t}$ by $\nu$, the associated Laplace exponent and mean at the unite time by

$$
\Phi(u):=\int_{0}^{\infty}\left(1-e^{-u y}\right) \nu(\mathrm{d} y), \quad \mu_{Z}:=\mathbb{E}\left[Z_{1}\right]=\int_{0}^{\infty} y \nu(\mathrm{d} y), \quad u>0
$$

the Laplace transform, mean of self-exciting jump sizes and a constant respectively by

$$
\hat{g}(u):=\int_{0}^{\infty} e^{-u y} \mathrm{~d} G(y), \quad \mu_{G}:=\int_{0}^{\infty} y \mathrm{~d} G(y), \quad \eta:=\delta-\mu_{G} .
$$

They are all assumed to be finite. In addition, we denote the $(i+1)^{\text {th }}$ interarrival time by

$$
\tau_{i+1}:=T_{i+1}-T_{i}, \quad i=0,1,2, \ldots, \quad T_{0}=0,
$$

and the cumulative intensity process at time $t$ by $\Lambda_{t}:=\int_{0}^{t} \lambda_{u} \mathrm{~d} u$.

One may be interested in their basic distributional properties such as means and Laplace transforms, and we provide some brief results for them in Appendix A with proofs. In particular, the conditional expectation of point process is provided here in Proposition 2.1, as it will be used later as a simple and general benchmark for numerically validating our newly-developed simulation al- 
gorithms.

Proposition 2.1 (Conditional Expectation of Point Process). The expectation of $N_{t+s}$ conditional on $N_{t}$ and $\lambda_{t}$ is given by

$$
\mathbb{E}\left[N_{t+s} \mid N_{t}, \lambda_{t}\right]=\left\{\begin{array}{ll}
N_{t}+\frac{\varrho \mu_{Z}}{\eta} s+\left(\lambda_{t}-\frac{\varrho \mu_{Z}}{\eta}\right) \frac{1-e^{-\eta s}}{\eta}, & \eta \neq 0, \\
N_{t}+\lambda_{t} s+\frac{1}{2} \varrho \mu_{Z} s^{2}, & \eta=0,
\end{array} \quad s>0 .\right.
$$

\section{General Framework for Exact Simulation}

In this section, we outline an exact simulation framework based on exact distributional decomposition for a general point process of Lévy-driven non-Gaussian OU intensity with and without self-exciting jumps as defined in Definition 2.2 and 2.1, respectively. The entire simulation scheme can be decomposed into three major steps:

1. Conditional on the current arrival time $T_{i}$ and the associated intensity level $\lambda_{T_{i}}$, generate the next interarrival time $\tau_{i+1}$;

2. Further conditional on the realisation of this interarrival time $\tau_{i+1}$, generate the pre-jump intensity level $\lambda_{T_{i}+\tau_{i+1}^{-}}$right before the next arrival time $T_{i+1}=T_{i}+\tau_{i+1}$;

3. Add a self-exciting jump size $X_{i+1}$ upon the intensity process $\lambda_{t}$ and one unit in the point process $N_{t}$ both at the next arrival time $T_{i+1}=T_{i}+\tau_{i+1}$.

By recursively implementing the three steps above, a full path of the point process $N_{t}$ in any time horizon can be exactly produced without bias. A graphical illustration for this proposed algorithm design is provided in Figure 2.

The third step indeed is straightforward. In particular, if $X_{i+1} \equiv 0$ for any $i$, then, it corresponds to the version without self-exciting jumps. In order to execute the first two steps, we have first to further investigate the joint distributional properties of the next interarrival time $\tau_{i+1}$ and the next pre-jump intensity level $\lambda_{T_{i}+\tau_{i+1}^{-}}$, which can be characterised by the conditional joint transform as below.

Theorem 3.1 (Joint Transform of Pre-jump Intensity and Cumulative Intensity). Conditional on the intensity level $\lambda_{T_{i}}$ at the $i^{\text {th }}$ arrival time $T_{i}$, the joint transform of $\left(\lambda_{T_{i}+\tau^{-}}, \Lambda_{T_{i}+\tau}-\Lambda_{T_{i}}\right)$ for any given period $\tau \in\left(0, \tau_{i+1}\right)$ is given by

$$
\mathbb{E}\left[e^{-v \lambda_{T_{i}+\tau^{-}}} e^{-\left(\Lambda_{T_{i}+\tau^{-}}-\Lambda_{T_{i}}\right)} \mid \lambda_{T_{i}}\right]
$$



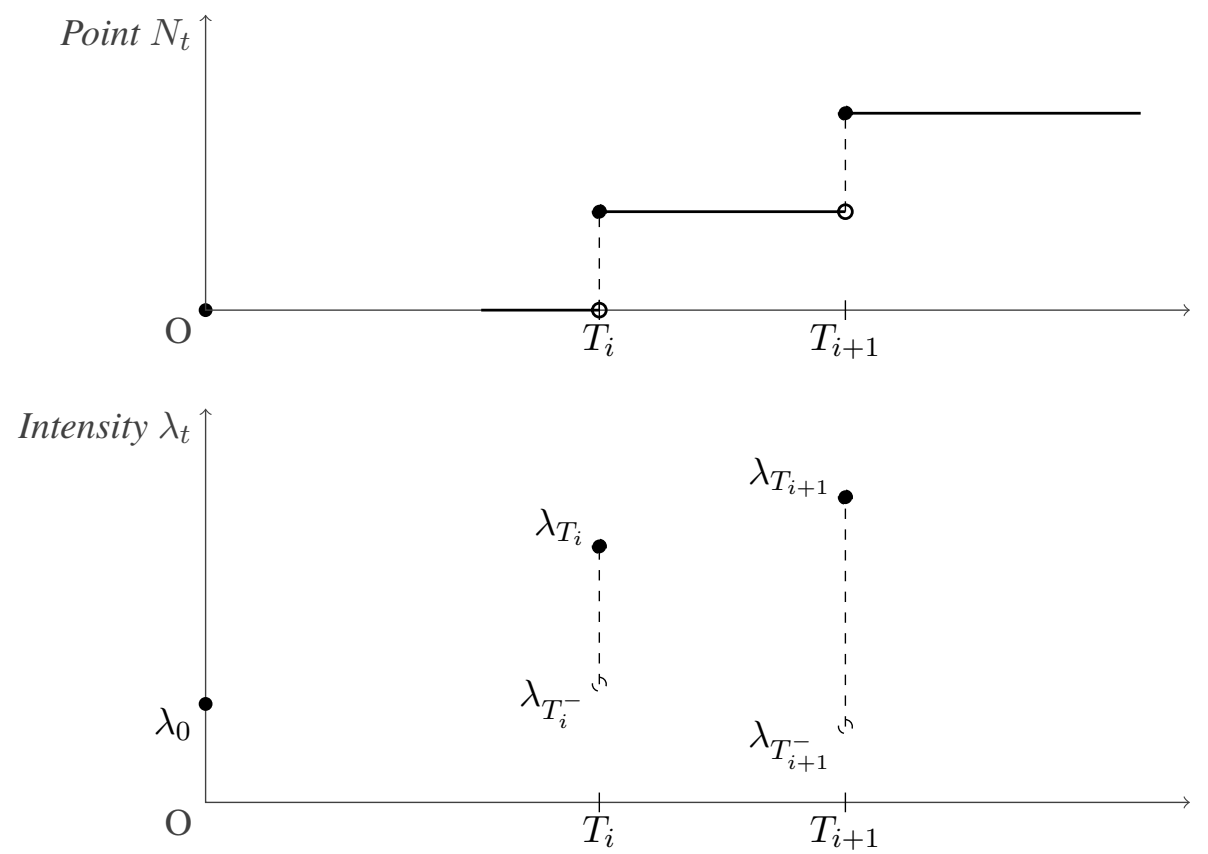

Figure 2: Illustration on the exact simulation procedures for a path of point process $N_{t}$ and the skeleton of its intensity process $\lambda_{t}$ around the period $\left[T_{i}, T_{i+1}\right]$

$$
=\exp \left(-\left[\frac{1}{\delta}+\left(v-\frac{1}{\delta}\right) w\right] \lambda_{T_{i}}-\varrho \int_{v}^{\frac{1}{\delta}+\left(v-\frac{1}{\delta}\right) w} \frac{\Phi(u)}{1-\delta u} \mathrm{~d} u\right), \quad \tau \in\left(0, \tau_{i+1}\right)
$$

where $w:=e^{-\delta \tau}$.

Proof. Given the $i^{\text {th }}$ arrival time $T_{i}$, the infinitesimal generator of $(\Lambda, \lambda, t)$ within the period $t \in$ $\left[T_{i}, T_{i}+\tau_{i+1}\right)$ acting on any function $f(\Lambda, \lambda, t)$ within its domain $\Omega(\mathcal{A})$ is given by

$$
\mathcal{A} f(\Lambda, \lambda, t)=\frac{\partial f}{\partial t}-\delta \lambda \frac{\partial f}{\partial \lambda}+\lambda \frac{\partial f}{\partial \Lambda}+\varrho\left\{\int_{0}^{\infty}[f(\Lambda, \lambda+y, t)-f(\Lambda, \lambda, t)] \nu(\mathrm{d} y)\right\} .
$$

Consider a function

$$
f(\Lambda, \lambda, t)=e^{-\tilde{v} \Lambda} e^{-\lambda A(t)} e^{R(t)}, \quad \tilde{v} \in \mathbb{R}^{+},
$$

where $A(t)$ and $R(t)$ are deterministic and differentiable functions with respect to $t$. Substituting (3.3) into (3.2) and setting $\mathcal{A} f=0$, we have

$$
-\lambda A^{\prime}(t)+R^{\prime}(t)+\delta \lambda A(t)-\tilde{v} \lambda-\varrho \Phi(A(t))=0 .
$$

Since this equation holds for any $\lambda$ and $\Lambda$, it is equivalent to the equations

$$
A^{\prime}(t)=\delta A(t)-\tilde{v}, \quad R^{\prime}(t)=\varrho \Phi(A(t)) .
$$


Hence, for any time $t \in\left[T_{i}, T_{i}+\tau_{i+1}\right)$, we have

$$
A(t)=k e^{\delta t}-\tilde{v} \frac{e^{\delta t}-1}{\delta}, \quad R(t)=\varrho \int_{0}^{t} \Phi\left(k e^{\delta s}-\tilde{v} \frac{e^{\delta s}-1}{\delta}\right) \mathrm{d} s, \quad k \in \mathbb{R}^{+}
$$

By the basic property of infinitesimal generator (Dassios and Embrechts, 1989), we have the martingale

$$
e^{-\tilde{v} \Lambda_{t}} \exp \left(-\left(k e^{\delta t}-\tilde{v} \frac{e^{\delta t}-1}{\delta}\right) \lambda_{t}+\varrho \int_{0}^{t} \Phi\left(k e^{\delta s}-\tilde{v} \frac{e^{\delta s}-1}{\delta}\right) \mathrm{d} s\right) .
$$

Setting $\tilde{v}=1$ and $A\left(T_{i}+\tau^{-}\right)=v$ for any $\tau \in\left(0, \tau_{i+1}\right)$ and using the martingale property, we have

$$
\begin{aligned}
& \mathbb{E}\left[e^{-v \lambda_{T_{i}+\tau^{-}}} e^{-\left(\Lambda_{T_{i}+\tau}-\Lambda_{T_{i}}\right)} \mid \lambda_{T_{i}}\right] \\
= & \exp \left(-\left[\frac{1}{\delta}+\left(v-\frac{1}{\delta}\right) w\right] \lambda_{T_{i}}-\varrho \int_{0}^{\tau} \Phi\left(\frac{1}{\delta}+\left(v-\frac{1}{\delta}\right) e^{-\delta s}\right) \mathrm{d} s\right) .
\end{aligned}
$$

Hence, we can immediately obtain (3.1) by the change of variable $u=\frac{1}{\delta}+\left(v-\frac{1}{\delta}\right) e^{-\delta s}$.

Theorem 3.1 provides us with a crucial tool for further investigating the distributional properties of the interarrival time $\tau_{i+1}$ and the pre-jump intensity level $\lambda_{T_{i}+\tau_{i+1}^{-}}$, jointly and separately, which later leads to their efficient algorithms for exact simulation as follows. Note that, however, the main mathematical contribution of this paper is the exact distributional decomposition for our new point processes via Theorem 3.1 rather than deriving the transform in Theorem 3.1.

\subsection{Exact Simulation of Interarrival Time}

Let us first outline how to simulate the interarrival time. Given the intensity level $\lambda_{T_{i}}$ at the $i^{\text {th }}$ arrival time $T_{i}$, interestingly, the $(i+1)^{\text {th }}$ interarrival time $\tau_{i+1}$ can be exactly expressed as the minimum of two much simpler random variables $V_{T_{i}}^{*}$ and $V^{*}$ where

1. $V_{T_{i}}^{*}$ is a defective random variable, which can be directly generated by an explicit inverse transform;

2. $V^{*}$ is a well-defined random variable, which can be exactly simulated by a simplified version of the classical thinning scheme (Lewis and Shedler, 1979).

Algorithm 3.1 (Exact Simulation of Interarrival Time). Conditional on the intensity level $\lambda_{T_{i}}$, the 
next interarrival time $\tau_{i+1}$ can be exactly simulated via

$$
\tau_{i+1} \stackrel{\mathcal{D}}{=} \begin{cases}V^{*} \wedge V_{T_{i}}^{*}, & D_{i}>0 \\ V^{*}, & D_{i}<0\end{cases}
$$

where

- $D_{i}$ is simulated via

$$
D_{i}:=1+\frac{\delta}{\lambda_{T_{i}}} \ln U_{1}, \quad U_{1} \sim U[0,1]
$$

- $V_{T_{i}}^{*}$ is a simple defective random variable with $\operatorname{Pr}\left\{V_{T_{i}}^{*}=\infty\right\}=\exp \left(-\frac{1}{\delta} \lambda_{T_{i}}\right)$,

$$
V_{T_{i}}^{*} \stackrel{\mathcal{D}}{=}-\frac{1}{\delta} \ln D_{i}, \quad D_{i}>0
$$

- $V^{*}$ is the first arrival time of a non-homogeneous Poisson process with the rate function

$$
\zeta_{t}:=\varrho \Phi\left(G_{0}(t)\right), \quad G_{0}(u):=\frac{1-e^{-\delta u}}{\delta}, \quad u \geq 0
$$

and it can be exactly simulated via the simplified thinning scheme of Algorithm 3.2.

Proof. Setting $v=0$ in (3.1) of Theorem 3.1, we have

$$
\operatorname{Pr}\left\{\tau_{i+1}>\tau \mid \lambda_{T_{i}}\right\}=\mathbb{E}\left[e^{-\left(\Lambda_{T_{i}+\tau}-\Lambda_{T_{i}}\right)} \mid \lambda_{T_{i}}\right]=\operatorname{Pr}\left\{V^{*}>\tau\right\} \times \operatorname{Pr}\left\{V_{T_{i}}^{*}>\tau\right\},
$$

where

$$
\operatorname{Pr}\left\{V^{*}>\tau\right\}=\exp \left(-\varrho \int_{0}^{\tau} \Phi\left(G_{0}(u)\right) \mathrm{d} u\right), \quad \operatorname{Pr}\left\{V_{T_{i}}^{*}>\tau\right\}=e^{-G_{0}(\tau) \lambda_{T_{i}}}
$$

This implies that, the next interarrival time $\tau_{i+1}$ conditional on the current intensity level $\lambda_{T_{i}}$ can be expressed as the minimum of two independent random variables $V^{*}$ and $V_{T_{i}}^{*}$. Note that, $V_{T_{i}}^{*}$ is a defective random variable that has a mass probability at the infinity, since the $\mathrm{CDF}$ of $V_{T_{i}}^{*}$ is

$$
F_{V_{T_{i}}^{*}}(\tau)=1-e^{-G_{0}(\tau) \lambda_{T_{i}}},
$$

with $F_{V_{T_{i}}^{*}}(\infty)=1-\exp \left(-\frac{1}{\delta} \lambda_{T_{i}}\right)<1$, and the density $f_{V_{T_{i}}^{*}}(\tau)>0$ for any $\tau>0$. Obviously, if $D_{i}>0$, then $V_{T_{i}}^{*}$ can be exactly simulated using the explicit inverse transform (3.5). Whereas $V^{*}$ can be interpreted as the first arrival time from a non-homogeneous Poisson process, and it can be exactly simulated via Algorithm 3.2 as below. 
Algorithm 3.2 (Simplified Thinning Scheme). $V^{*}$ can be exactly simulated by the following steps:

1. Initialise the candidate time $\tilde{t}=0$;

2. Generate an exponential random variable $E^{*} \sim \operatorname{Exp}\left(\zeta_{\infty}\right)$ where

$$
\zeta_{\infty}:=\lim _{t \rightarrow \infty} \zeta_{t}=\varrho \Phi\left(\frac{1}{\delta}\right)
$$

and set $\tilde{t}=\tilde{t}+E^{*}$;

3. Generate a uniform random variable $U_{2} \sim U[0,1]$,

- if $U_{2} \leq \zeta_{\tilde{t}} / \zeta_{\infty}$, then, accept this candidate by setting $V^{*}=\tilde{t}$;

- if $U_{2}>\zeta_{\tilde{t}} / \zeta_{\infty}$, then, reject this candidate, and go back to Step 2 and continue.

Proof. Since $\zeta_{t}$ in (3.6) is a strictly increasing and concave function of time $t$ with the initial value $\zeta_{0}=0$ at time $t=0$, the maximum level is $\zeta_{\infty}$. Then, the algorithm above actually is a simplified version of the classical thinning scheme (Lewis and Shedler, 1979) where only the first arrival time within the period of $[0, t]$ is recorded.

\subsection{Exact Simulation of Pre-jump Intensity Level}

Conditional the realisation of interarrival time $\tau_{i+1}$ as generated by Algorithm 3.1, the Laplace transform of the next pre-jump intensity level $\lambda_{T_{i}+\tau_{i+1}^{-}}$is provided as follows.

Theorem 3.2 (Laplace Transform of Pre-jump Intensity). Conditional on the intensity level $\lambda_{T_{i}}$ and the $(i+1)^{\text {th }}$ interarrival time $\tau_{i+1}$, the Laplace transform of pre-jump intensity level $\lambda_{T_{i}+\tau_{i+1}^{-}}$ is given by

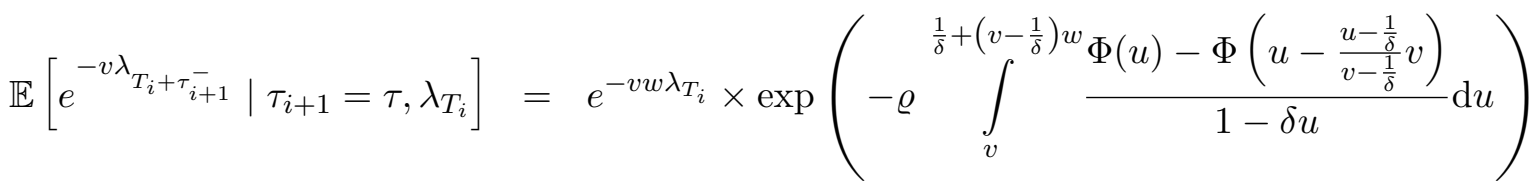

$$
\begin{aligned}
& \times \frac{\frac{\varrho}{\delta} \int_{0}^{\infty} e^{-v s} \int_{s}^{\frac{s}{w}} e^{\frac{s}{\delta}} e^{-\frac{y}{\delta}} \nu(\mathrm{d} y) \mathrm{d} s+w \lambda_{T_{i}}}{\frac{\varrho}{\delta} \int_{0}^{\infty} \int_{s}^{\frac{s}{w}} e^{\frac{s}{\delta}} e^{-\frac{y}{\delta}} \nu(\mathrm{d} y) \mathrm{d} s+w \lambda_{T_{i}}} .
\end{aligned}
$$

Proof. Note that, the density function of the $(i+1)^{\text {th }}$ interarrival time conditional on the intensity 
level $\lambda_{T_{i}}$ is

$$
\operatorname{Pr}\left\{\tau_{i+1} \in \mathrm{d} \tau \mid \lambda_{T_{i}}\right\}=\mathbb{E}\left[\lambda_{T_{i}+\tau^{-}} \exp \left(-\int_{T_{i}}^{T_{i}+\tau} \lambda_{u} \mathrm{~d} u\right) \mid \lambda_{T_{i}}\right] \mathrm{d} \tau
$$

which implies that,

$$
\mathbb{E}\left[e^{-v \lambda_{T_{i}+\tau_{i+1}^{-}}} \mathbf{1}_{\left\{\tau_{i+1} \in \mathrm{d} \tau\right\}} \mid \lambda_{T_{i}}\right]=\mathbb{E}\left[\lambda_{T_{i}+\tau^{-}} e^{-v \lambda_{T_{i}+\tau^{-}}} \exp \left(-\int_{T_{i}}^{T_{i}+\tau} \lambda_{u} \mathrm{~d} u\right) \mid \lambda_{T_{i}}\right] \mathrm{d} \tau
$$

Hence, we have

$$
\mathbb{E}\left[e^{-v \lambda_{T_{i}+\tau_{i+1}^{-}}} \mid \tau_{i+1}=\tau, \lambda_{T_{i}}\right]=\frac{\mathbb{E}\left[\lambda_{T_{i}+\tau^{-}} e^{-v \lambda_{T_{i}+\tau^{-}}} e^{-\left(\Lambda_{T+\tau}-\Lambda_{T_{i}}\right)} \mid \lambda_{T_{i}}\right]}{\mathbb{E}\left[\lambda_{T_{i}+\tau^{-}} e^{-\left(\Lambda_{\left.T_{i}+\tau^{-}-\Lambda_{T_{i}}\right)}\right)} \mid \lambda_{T_{i}}\right]} .
$$

The numerator of (3.10) can be obtained by differentiating the joint transform (3.1) w.r.t. $v$, i.e.,

$$
\begin{aligned}
& \mathbb{E}\left[\lambda_{T_{i}+\tau^{-}} e^{-v \lambda_{T_{i}+\tau^{-}}} e^{-\left(\Lambda_{T_{i}+\tau^{-}}-\Lambda_{T_{i}}\right)} \mid \lambda_{T_{i}}\right] \\
= & -\frac{\partial}{\partial v} \mathbb{E}\left[e^{-v \lambda_{T_{i}+\tau^{-}}} e^{-\left(\Lambda_{T_{i}+\tau^{-}}-\Lambda_{T_{i}}\right)} \mid \lambda_{T_{i}}\right] \\
= & -\frac{\partial}{\partial v}\left[\exp \left(-\left[\frac{1}{\delta}+\left(v-\frac{1}{\delta}\right) e^{-\delta \tau}\right] \lambda_{T_{i}}\right) \times \exp \left(-\varrho \int_{0}^{\tau} \Phi\left(\frac{1}{\delta}+\left(v-\frac{1}{\delta}\right) e^{-\delta u}\right) \mathrm{d} u\right)\right] \\
= & -\frac{\partial}{\partial v}\left[e^{\left.-G_{v}(\tau) \lambda_{T_{i}} \times e^{-\varrho F_{v}(\tau)}\right]}\right. \\
= & -\left[\varrho \frac{\partial}{\partial v} F_{v}(\tau)+\frac{\partial}{\partial v} G_{v}(\tau)\right] \times e^{-G_{v}(\tau) \lambda_{T_{i}}} e^{-\varrho F_{v}(\tau)},
\end{aligned}
$$

where

$$
G_{v}(u):=\frac{1}{\delta}+\left(v-\frac{1}{\delta}\right) e^{-\delta u}, \quad F_{v}(\tau):=\int_{0}^{\tau} \Phi\left(G_{v}(u)\right) \mathrm{d} u
$$

and

$$
\begin{aligned}
\frac{\partial}{\partial v} G_{v}(\tau) & =-\lambda_{T_{i}} e^{-\delta \tau} \\
\frac{\partial}{\partial v} F_{v}(\tau) & =\int_{0}^{\tau} \int_{0}^{\infty} y e^{-\delta u} e^{-\left[\frac{1}{\delta}+\left(v-\frac{1}{\delta}\right) e^{-\delta u}\right] y} \nu(\mathrm{d} y) \mathrm{d} u .
\end{aligned}
$$

Note that, $\nu$ is the Lévy measure for a general BDLP $Z_{t}$, therefore, we have

$$
\mathbb{E}\left[e^{-v \lambda_{T_{i}+\tau_{i+1}^{-}}} \mid \tau_{i+1}=\tau, \lambda_{T_{i}}\right]
$$




$$
\begin{aligned}
& =\frac{\left(\varrho \int_{0}^{\tau} \int_{0}^{\infty} y e^{-\delta u} e^{-\left[\frac{1}{\delta}+\left(v-\frac{1}{\delta}\right) e^{-\delta u}\right] y} \nu(\mathrm{d} y) \mathrm{d} u+w \lambda_{T_{i}}\right) \times e^{-G_{v}(\tau) \lambda_{T_{i}}} e^{-\varrho F_{v}(\tau)}}{\left(\varrho \int_{0}^{\tau} \int_{0}^{\infty} y e^{-\delta u} e^{-\frac{1}{\delta}\left(1-e^{-\delta u}\right) y} \nu(\mathrm{d} y) \mathrm{d} u+w \lambda_{T_{i}}\right) \times e^{-G_{0}(\tau) \lambda_{T_{i}}} e^{-\varrho F_{0}(\tau)}} \\
& =\frac{\varrho \int_{0}^{\tau} \int_{0}^{\infty} y e^{-\delta u} e^{-\left[\frac{1}{\delta}+\left(v-\frac{1}{\delta}\right) e^{-\delta u}\right] y} \nu(\mathrm{d} y) \mathrm{d} u+w \lambda_{T_{i}}}{\varrho \int_{0}^{\tau} \int_{0}^{\infty} y e^{-\delta u} e^{-\frac{1}{\delta}\left(1-e^{-\delta u}\right) y} \nu(\mathrm{d} y) \mathrm{d} u+w \lambda_{T_{i}}} \times \frac{\mathbb{E}\left[e^{-v \lambda_{T_{i}+\tau^{-}}} e^{-\left(\Lambda_{T_{i}+\tau^{-}}-\Lambda_{T_{i}}\right)} \mid \lambda_{T_{i}}\right]}{\mathbb{E}\left[e^{-\left(\Lambda_{\left.T_{i}+\tau^{-}-\Lambda_{T_{i}}\right)}\right)} \mid \lambda_{T_{i}}\right]} .
\end{aligned}
$$

The first term of (3.11) can be calculated more explicitly as

$$
\begin{aligned}
& \varrho \int^{\tau} \int_{0}^{\infty} y e^{-\delta u} e^{-\left[\frac{1}{\delta}+\left(v-\frac{1}{\delta}\right) e^{-\delta u}\right] y} \nu(\mathrm{d} y) \mathrm{d} u+w \lambda_{T_{i}} \\
& \begin{array}{ll}
0 \quad 0 \\
\hline
\end{array} \\
& \varrho \int_{0}^{\tau} \int_{0}^{\infty} y e^{-\delta u} e^{-\frac{1}{\delta}\left(1-e^{-\delta u}\right) y} \nu(\mathrm{d} y) \mathrm{d} u+w \lambda_{T_{i}} \\
& =\frac{\varrho \int_{v}^{\frac{1}{\delta}+\left(v-\frac{1}{\delta}\right) w} \int_{0}^{\infty} y \frac{u-\frac{1}{\delta}}{v-\frac{1}{\delta}} e^{-u y} \nu(\mathrm{d} y) \frac{\mathrm{d} u}{1-\delta u}+w \lambda_{T_{i}}}{\varrho \int_{v}^{\frac{1}{\delta}+\left(v-\frac{1}{\delta}\right) w} \int_{0}^{\infty} y \frac{u-\frac{1}{\delta}}{v-\frac{1}{\delta}} e^{-\left(u-\frac{u-\frac{1}{\delta}}{v-\frac{1}{\delta}} v\right) y} \nu(\mathrm{d} y) \frac{\mathrm{d} u}{1-\delta u}+w \lambda_{T_{i}}} \\
& =\frac{\frac{\varrho}{1-\delta v} \int_{v}^{\frac{1}{\delta}+\left(v-\frac{1}{\delta}\right) w} \int_{0}^{\infty} y e^{-\left(u-\frac{1}{\delta}\right) y} e^{-\frac{y}{\delta}} \nu(\mathrm{d} y) \mathrm{d} u+w \lambda_{T_{i}}}{\frac{\varrho}{1-\delta v} \int_{v}^{\frac{1}{\delta}+\left(v-\frac{1}{\delta}\right) w} \int_{0}^{\infty} y e^{-\left(u-\frac{1}{\delta}-\frac{u-\frac{1}{\delta}}{v-\frac{1}{\delta}} v\right) y} e^{-\frac{y}{\delta}} \nu(\mathrm{d} y) \mathrm{d} u+w \lambda_{T_{i}}} \\
& =\frac{-\frac{\varrho}{\delta} \int_{1-\delta v}^{(1-\delta v) w} \int_{0}^{\infty} y e^{\frac{(1-\delta v) z y}{\delta}} e^{-\frac{y}{\delta}} \nu(\mathrm{d} y) \mathrm{d} z+w \lambda_{T_{i}}}{(1-\delta v) w \infty} \\
& -\frac{\varrho}{\delta} \int_{1-\delta v}^{(1-\delta v) w} \int_{0}^{\infty} y e^{\frac{z y}{\delta}} e^{-\frac{y}{\delta}} \nu(\mathrm{d} y) \mathrm{d} z+w \lambda_{T_{i}} \\
& \frac{\varrho}{\delta} \int_{0}^{\infty} e^{-v s} \int_{s}^{\frac{s}{w}} e^{\frac{s}{\delta}} e^{-\frac{y}{\delta}} \nu(\mathrm{d} y) \mathrm{d} s+w \lambda_{T_{i}} \\
& =\frac{0}{\frac{\varrho}{\delta} \int_{0}^{\infty} \int_{s}^{\frac{s}{w}} e^{\frac{s}{\delta}} e^{-\frac{y}{\delta}} \nu(\mathrm{d} y) \mathrm{d} s+w \lambda_{T_{i}}}
\end{aligned}
$$

As the denominator of the second term of (3.11) can be also obtained nicely by setting $v=0$ in 
the joint transform (3.1), i.e.,

$$
\mathbb{E}\left[e^{-\left(\Lambda_{T_{i}+\tau}-\Lambda_{T_{i}}\right)} \mid \lambda_{T_{i}}\right]=\exp \left(-\frac{1-w}{\delta} \lambda_{T_{i}}-\varrho \int_{v}^{\frac{1}{\delta}+\left(v-\frac{1}{\delta}\right) w} \frac{\left(u-\frac{u-\frac{1}{\delta}}{v-\frac{1}{\delta}} v\right)}{1-\delta u} \mathrm{~d} s\right)
$$

So, the second term of (3.11) can expressed by

$$
\begin{aligned}
& \frac{\mathbb{E}\left[e^{-v \lambda_{T_{i}+\tau^{-}}} e^{-\left(\Lambda_{T_{i}+\tau}-\Lambda_{T_{i}}\right)} \mid \lambda_{T_{i}}\right]}{\mathbb{E}\left[e^{-\left(\Lambda_{T_{i}+\tau}-\Lambda_{T_{i}}\right)} \mid \lambda_{T_{i}}\right]} \\
= & \frac{\exp \left(-\left[\frac{1}{\delta}+\left(v-\frac{1}{\delta}\right) w\right] \lambda_{T_{i}}\right) \exp \left(-\varrho \int_{v}^{\frac{1}{\delta}+\left(v-\frac{1}{\delta}\right) w} \frac{\Phi(u)}{1-\delta u} \mathrm{~d} u\right)}{\exp \left(-\frac{1}{\delta}(1-w) \lambda_{T_{i}}\right) \exp \left(-\varrho \int_{v}^{\frac{1}{\delta}+\left(v-\frac{1}{\delta}\right) w} \frac{\left(u-\frac{u-\frac{1}{\delta}}{v-\frac{1}{\delta}} v\right)}{1-\delta u} \mathrm{~d} u\right)} \\
= & \left.e^{-v w \lambda_{T_{i}} \times \exp \left(-\varrho \int_{v}^{\frac{1}{\delta}+\left(v-\frac{1}{\delta}\right) w} \Phi(u)-\Phi\left(u-\frac{u-\frac{1}{\delta}}{v-\frac{1}{\delta}} v\right)\right.} \mathrm{d} u\right) .
\end{aligned}
$$

Finally, we obtain (3.9) immediately by combining the results from (3.12) and (3.13).

Apparently, given the $i^{\text {th }}$ arrival time $T_{i}$ and the $(i+1)^{\text {th }}$ interarrival time $\tau_{i+1}$, the pre-jump intensity level $\lambda_{T_{i}+\tau_{i+1}^{-}}$can be simulated by the numerical inversion of Laplace transform (3.9) for any Lévy-driven contagion process once the associated Lévy measure $\nu$ (and Laplace exponent $\Phi$ ) are specified. Indeed, exact simulation for stochastic processes based on the numerical inversion of Laplace or Fourier transform has been widely adopted in the literature, see Broadie and Kaya (2006), Glasserman and Liu (2010), Chen et al. (2012), Cai et al. (2017) and Kang et al. (2017).

However, for some subclasses such as the very popular specifications of gamma and tempered stable BDLPs, quite remarkably, based on Theorem 3.2 the pre-jump intensity level can be exactly decomposed into several simple elements, each of which can be easily simulated exactly without any numerical inversion procedure. In fact, this exact decomposition approach appropriately breaks the Lévy measure of subordinator, and thereby it can be achieved by developing an exact distributional decomposition through Laplace-transform representations. In this paper, our focus is mainly on this decomposition approach, as it leads to a very efficient simulation algorithm for exactly sampling the whole point process, and more importantly, it does not involve additional discretisation or truncation errors which are inevitable in the numerical inversion approach. We will present our discovery based on the decomposition approach in much more details later in Section 4. 


\subsection{Exact Simulation of Self-exciting Jumps}

Based on our key results of Algorithm 3.1 for the interarrival time $\tau_{i+1}$ and Theorem 3.2 for the associated pre-jump intensity level $\lambda_{T_{i}+\tau_{i+1}^{-}}$, now, it is straightforward to further integrate the selfexciting jumps as the final step.

Algorithm 3.3 (Exact Simulation of Self-exciting Jumps). Conditional on $\left(\lambda_{T_{i}}, T_{i}\right)$ for any step index $i \in \mathbb{N}^{+}$, the next self-exciting jumps occurring simultaneously in the intensity process and the point process can be exactly simulated via the following steps:

1. Simulate the $(i+1)^{\text {th }}$ interarrival time $\tau_{i+1}$ by thinning via Algorithm 3.1;

2. Set the $(i+1)^{\text {th }}$ arrival time by $T_{i+1}=T_{i}+\tau_{i+1}$;

3. Simulate the $(i+1)^{\text {th }}$ pre-jump intensity $\lambda_{T_{i+1}^{-}}$by numerical inversion or exact decomposition for its Laplace transform in Theorem 3.2;

4. Add a self-exciting jump of size $X_{i+1}$ to the intensity process at the $(i+1)^{\text {th }}$ arrival time $T_{i+1}$, i.e.

$$
\lambda_{T_{i+1}}=\lambda_{T_{i+1}^{-}}+X_{i+1}
$$

5. Add one unit to the point process at the $(i+1)^{\text {th }}$ arrival time $T_{i+1}$, i.e. $N_{T_{i+1}}=N_{T_{i+1}^{-}}+1$.

By recursively implementing Algorithm 3.3, the skeleton of any non-Gaussian OU intensity process $\lambda_{t}$ and the associated full path of point process $N_{t}$ in continuous time can be exactly generated. Moreover, there is almost no restriction on the size of self-exciting jump, $X_{i+1}$. It is very flexible, as long as it would not overshoot the zero bound: it could be a constant, or, a random variable having a highly general dependency on the past information before and at the arrival time $T_{i}$.

Overall, the whole process can be decomposed into interarrival times, pre-jump intensity levels and self-exciting jumps. In general, each of pre-jump intensity levels in Step 3 can be simulated in general by numerically inverting (3.9) using the Fourier inversion technique, once we specify a subordinator for the BDLP $Z_{t}$. However, for some subclasses, even the numerical inversion can be avoided. That is, the pre-jump intensity allows a further exact distributional decomposition, which leads to an exact simulation algorithm without numerical inversion. The resulting scheme thereby has no bias or truncation errors. 


\section{Typical Examples: Gamma and Tempered Stable Contagion Mod- els}

For model implementation, one needs to further specify the BDLP in an explicit form. Probably the most widely used and representative Lévy subordinators in the literature are gamma process and tempered stable (TS) subordinator ${ }^{6}$. More precisely, they are two typical examples of Lévy processes with stationary, independent and non-negative increments starting at 0 with finite variation and infinite activity ${ }^{7}$, which behave very differently from the trivial case of compound Poisson process. Due to this nature, they are impossible to be simulated exactly by some traditional methods on discretising sample paths. We provide the definitions for them using Lévy measures (4.2) and (4.5) in Definition 4.1 and 4.2, respectively.

Definition 4.1 (Gamma Distribution and Gamma Process). The gamma distribution with shape parameter $a$ and rate parameter $b$, denoted by $\operatorname{Gamma}(a, b)$, has the density function

$$
f_{\operatorname{Gamma}(a, b)}(s)=\frac{b^{a}}{\Gamma(a)} s^{a-1} e^{-b s}, \quad s>0, \quad a, b>0,
$$

where $\Gamma(\cdot)$ is the gamma function, i.e. $\Gamma(u):=\int_{0}^{\infty} s^{u-1} e^{-s} \mathrm{~d}$ s. gamma process $\left\{G_{t}, t \geq 0\right\}$ is a pure-jump increasing Lévy process with independently gamma distributed increments satisfying $G_{1} \sim \operatorname{Gamma}(a, b), G_{0}=0$, and it has Lévy measure

$$
\nu(\mathrm{d} s)=a s^{-1} e^{-b s} \mathrm{~d} s
$$

and the Laplace exponent

$$
\Phi(u)=a \ln \left(1+\frac{u}{b}\right)
$$

and the mean at unit time

$$
\mu_{Z}=\mathbb{E}\left[Z_{1}\right]=\frac{a}{b}
$$

The gamma distribution, as a well-known right-skewed distribution, is approximated by a normal distribution when its mean is large, and it has many computational conveniences ${ }^{8}$ and thereby is a very useful building block as the risk driver. Therefore, we provide applications for the gammacontagion model later in Section 6.

\footnotetext{
${ }^{6}$ The term "tempered stable" in the context sometimes refers to "exponential tilted stable", see Sato (1999) and Rosiński (2007) for more information.

${ }^{7}$ Infinite activity means that there are infinitely many small jumps within any finite time interval, see some strong evidence from financial data in Li et al. (2008), Ait-Sahalia and Jacod (2009, 2011) and Lee and Hannig (2010).

${ }^{8} \mathrm{~A}$ well-known convenience, similar to the normal distribution, is that, if $\left\{Y_{i}\right\}_{i=1,2, \ldots, m}$ are i.i.d. random variables following a gamma distribution with mean $\mu_{Y}$ and variance $\sigma_{Y}^{2}$, then, the sum $\sum_{i=1}^{m} Y_{i}$ is gamma distributed with mean $m \mu_{Y}$ and variance $m \sigma_{Y}^{2}$.
} 
Definition 4.2 (Tempered Stable (TS) Distribution and Tempered Stable (TS) Subordinator). The positive tempered stable (TS) distribution, abbreviated as $T S(\alpha, \beta, \theta)$, is defined by its Lévy measure

$$
\nu(\mathrm{d} y)=\frac{\theta}{y^{\alpha+1}} e^{-\beta y} \mathrm{~d} y, \quad y \geq 0, \quad \alpha \in(0,1), \quad \beta, \theta \in \mathbb{R}^{+},
$$

with the Laplace exponent

$$
\Phi(u)=-\theta \Gamma(-\alpha)\left[(\beta+u)^{\alpha}-\beta^{\alpha}\right]
$$

the mean at unite time

$$
\mu_{Z}=\mathbb{E}\left[Z_{1}\right]=\theta \beta^{\alpha-1} \Gamma(1-\alpha),
$$

where $\alpha$ is the stability index, $\theta$ is the intensity parameter and $\beta$ is the tilting parameter. The tempered stable (TS) subordinator is a Lévy process $\left\{Z_{t}: t \geq 0\right\}$ such that $Z_{1} \sim T S(\alpha, \beta, \theta)$ for $0<\alpha<1$ and $\beta, \theta>0$.

In fact, tempered stable distribution with three parameters is a very general and flexible distribution. The stable index $\alpha$ determines the importance of small jumps for the process trajectories, the intensity parameter $\theta$ controls the intensity of jumps, and the tilting parameter $\beta$ determines the decay rate of large jumps. In particular, if $\alpha=\frac{1}{2}$, it reduces to a very important distribution, the inverse Gaussian (IG) distribution, which can be interpreted as the distribution of the first passage time of a Brownian motion to an absorbing barrier. So, this family of tempered stable subordinator in Definition 4.2 also covers the inverse Gaussian $(I G)$ subordinator as an important special case (Barndorff-Nielsen, 1997, 1998). Conventionally, the IG distribution is denoted by IG $\left(\mu_{\mathrm{IG}}, \lambda_{\mathrm{IG}}\right)$ where $\mu_{\mathrm{IG}}$ is the mean parameter and $\lambda_{\mathrm{IG}}$ is the rate parameter, see a detailed introduction for IG distributions in Chhikara and Folks (1989). The IG subordinator is a special TS subordinator such that the BDLP $Z_{t} \sim \mathrm{IG}\left(\frac{t}{c}, t^{2}\right)$ for any $c, t \in \mathbb{R}^{+}$, i.e.,

$$
\mathrm{IG}\left(\frac{t}{c}, t^{2}\right) \stackrel{\mathcal{D}}{=} \mathrm{TS}\left(\frac{1}{2}, \frac{c^{2}}{2}, \frac{t}{\sqrt{2 \pi}}\right)
$$

Many scholars adopted gamma, inverse Gaussian and tempered stable subordinators as the building blocks to further construct other useful stochastic processes, and there are tremendous relevant papers and work in the literature, see Barndorff-Nielsen and Shephard (2001a,b, 2003a), Cont and Tankov (2004), Kyprianou (2006), Schoutens and Cariboni (2010) and Li and Linetsky (2014) to name a few. Their main attractiveness is that, the resulting models could possess skewness and leptokurtosis marginally, and meanwhile they remain highly mathematically tractable. Our new models introduced in this paper can additionally incorporate the "contagion" property, which 
is also very desirable from the point of view for applications.

Exact Simulation of Interarrival Time For each case, the interarrival time can be simulated via the general algorithm of thinning scheme, Algorithm 3.2, by simply calculating $\zeta_{\infty}$ in (3.8) from (3.6) explicitly as

$$
\zeta_{\infty}= \begin{cases}\varrho a \ln \left(1+\frac{1}{\delta b}\right), & \text { for Gamma } \\ -\varrho \theta \Gamma(-\alpha)\left[\left(\beta+\frac{1}{\delta}\right)^{\alpha}-\beta^{\alpha}\right], & \text { for TS }\end{cases}
$$

Exact Simulation of Pre-jump Intensity The pre-jump intensity level conditional on the realisation of interarrival time is characterised by the Laplace transforms (3.9) in Theorem 3.2, with Lévy measures $\nu$ and Laplace exponents $\Phi$ specified by $(4.2,4.3)$ and $(4.5,4.6)$ for the gamma and TS cases, respectively. For both cases, the integral transforms of the pre-jump intensity levels actually can be broken into several simple elements.

The Laplace transform of pre-jump intensity level with three terms in (3.9) actually consists two parts: (3.12) and (3.13). Strikingly, based on Theorem 3.2, the first two terms of (3.9), i.e., (3.13), can be further exactly decomposed for the specified TS and gamma cases respectively as follows.

Algorithm 4.1 (Exact Simulation of Pre-jump Intensity Level for $\Gamma$-Contagion). For the $\Gamma$-contagion, conditional on the intensity level $\lambda_{T_{i}}$ and the realisation of the $(i+1)^{\text {th }}$ interarrival time $\tau_{i+1}=\tau$, the distribution of the $(i+1)^{\text {th }}$ pre-jump intensity level $\lambda_{T_{i}+\tau^{-}}$can be exactly decomposed by

$$
\lambda_{T_{i}+\tau^{-}} \mid \lambda_{T_{i}} \stackrel{\mathcal{D}}{=} w \lambda_{T_{i}}+\widetilde{\Gamma}+\widetilde{B} \times S+\sum_{j=1}^{\widetilde{N}} S_{j}
$$

where $\widetilde{\Gamma}, \widetilde{B}, S, \widetilde{N}$ and $\left\{S_{j}\right\}_{j=1,2, \ldots}$ are all independent of each other,

- $\widetilde{\Gamma}$ is a gamma random variable of

$$
\widetilde{\Gamma} \sim \operatorname{Gamma}\left(-\frac{a \varrho}{\delta} \ln w, \frac{\vartheta}{w}-\frac{1}{\delta}\right), \quad \vartheta:=b+\frac{1}{\delta}
$$

- $\widetilde{B}$ is a Bernoulli random variable taking 0 with probability $p_{1}$ and 1 with probability $p_{2}$, and

$$
p_{1}=\frac{w \lambda_{T_{i}}}{\frac{a \varrho}{\delta} C+w \lambda_{T_{i}}}, \quad p_{2}=\frac{\frac{a \varrho}{\delta} C}{\frac{a \varrho}{\delta} C+w \lambda_{T_{i}}}, \quad C:=\delta \ln \left(\frac{b \delta+1-w}{b \delta}\right)
$$


- $S$ is an exponential random variable of $S \sim \operatorname{Exp}\left(\vartheta W_{0}-\frac{1}{\delta}\right)$ and

$$
W_{0} \stackrel{\mathcal{D}}{=}\left[1-b \delta\left(e^{\frac{C}{\delta} U_{0}}-1\right)\right]^{-1}, \quad U_{0} \sim U[0,1]
$$

- $\widetilde{N}$ is a Poisson random variable of rate $\frac{a \varrho}{\delta} \vartheta C_{w}$ and

$$
C_{w}:=\int_{1}^{\frac{1}{w}} \frac{\ln u}{\vartheta u-\frac{1}{\delta}} \mathrm{d} u
$$

- $\left\{S_{j}\right\}_{j=1,2, \ldots}$ are i.i.d. with $S_{j} \sim \operatorname{Exp}\left(\vartheta W-\frac{1}{\delta}\right)$, and $W$ can be exactly simulated via the A/R scheme of Algorithm B.1.

Proof. In fact, Algorithm 4.1 is only an explicit specification of Theorem 3.2. Let us first calculate the first two terms of (3.9), i.e., (3.13), by

$$
\begin{aligned}
& \frac{\mathbb{E}\left[e^{-v \lambda_{T_{i}+\tau^{-}}} e^{-\left(\Lambda_{T_{i}+\tau^{-}}-\Lambda_{T_{i}}\right)} \mid \lambda_{T_{i}}\right]}{\mathbb{E}\left[e^{-\left(\Lambda_{T_{i}+\tau^{-}}-\Lambda_{T_{i}}\right)} \mid \lambda_{T_{i}}\right]} \\
= & e^{-v w \lambda_{T_{i}}} \times \exp \left(-\frac{a \varrho}{\delta} \ln \left(\frac{1}{w}\right) \int_{0}^{\infty}\left(1-e^{-v s}\right) s^{-1} e^{-\left(\frac{\vartheta}{w}-\frac{1}{\delta}\right) s} \mathrm{~d} s\right) \\
& \times \exp \left(-\frac{a \vartheta \varrho}{\delta} \int_{0}^{\infty}\left(1-e^{-v s}\right) \int_{1}^{\frac{1}{w}}\left(\vartheta u-\frac{1}{\delta}\right) e^{-\left(\vartheta u-\frac{1}{\delta}\right) s} \frac{\ln u}{\vartheta u-\frac{1}{\delta}} \mathrm{d} u \mathrm{~d} s\right) .
\end{aligned}
$$

Then, by calculating the whole equation (3.9) more explicitly, the conditional Laplace transform of the pre-jump intensity level $\lambda_{T_{i}+\tau^{-}}$can be decomposed into four parts:

$$
\begin{aligned}
& \mathbb{E}\left[e^{-v \lambda_{T_{i}+\tau_{i+1}^{-}}} \mid \tau_{i+1}=\tau, \lambda_{T_{i}}\right] \\
= & e^{-v w \lambda_{T_{i}}} \times \exp \left(-\frac{\varrho}{\delta} \ln \left(\frac{1}{w}\right) \int_{0}^{\infty}\left(1-e^{-v s}\right) s^{-1} e^{-\left(\frac{\theta}{w}-\frac{1}{\delta}\right) s} \mathrm{~d} s\right) \\
& \times \exp \left(-\frac{a \vartheta \varrho}{\delta} \int_{0}^{\infty}\left(1-e^{-v s}\right) \int_{1}^{\frac{1}{w}}\left(\vartheta u-\frac{1}{\delta}\right) e^{-\left(\vartheta u-\frac{1}{\delta}\right) s} \frac{\ln u}{\vartheta u-\frac{1}{\delta}} \mathrm{d} u \mathrm{~d} s\right) \\
& \times\left[\frac{\frac{a \varrho C}{\delta}}{\frac{a \varrho C}{\delta}+w \lambda_{T_{i}}} \int_{0}^{\infty} e^{-v s} \int_{1}^{\frac{1}{w}}\left(\vartheta u+\frac{1}{\delta}\right) e^{-\left(\vartheta u-\frac{1}{\delta}\right) s} \frac{1}{C\left(\vartheta u^{2}-\frac{1}{\delta} u\right)} \mathrm{d} u \mathrm{~d} s+\frac{w \lambda_{T_{i}}}{\frac{a \varrho C}{\delta}+w \lambda_{T_{i}}}\right] .
\end{aligned}
$$

This decomposition of (4.12) indicates that the conditional distribution of $\lambda_{T_{i}+\tau^{-}}$is the sum of four independent simple elements of (4.8): (1) one deterministic trend, (2) one random variable 
$\widetilde{B} \times S$, (3) one gamma random variable, and (4) one compound Poisson random variable. Note that, $\widetilde{B} \times S$ can be alternatively defined as just one single random variable by

$$
\widetilde{B} \times S \stackrel{\mathcal{D}}{=} \begin{cases}0, & \text { with probability } p_{1}=\frac{w \lambda_{T_{i}}}{\frac{a \varrho}{\delta} C+w \lambda_{T_{i}}}, \\ S \sim \operatorname{Exp}\left(\vartheta W_{0}-\frac{1}{\delta}\right), & \text { with probability } p_{2}=\frac{\frac{a \varrho}{\delta} C}{\frac{a \varrho}{\delta} C+w \lambda_{T_{i}}}\end{cases}
$$

The CDF of $W_{0}$ is

$$
F_{W_{0}}(u)=\frac{1}{C_{w}\left(a+\frac{1}{\delta}\right)} \ln \left(\frac{\left(a+\frac{1}{\delta}\right) u-\frac{1}{\delta}}{a}\right), \quad u \in\left[1, \frac{1}{w}\right]
$$

which can be inverted explicitly, so we have (4.10). The compound Poisson random variable $\sum_{j=1}^{\tilde{N}} S_{j}$ has the Laplace transform

$$
\exp \left(-\frac{a \vartheta \varrho}{\delta} C_{w} \int_{0}^{\infty}\left(1-e^{-v s}\right) \int_{1}^{\frac{1}{w}}\left(\vartheta u-\frac{1}{\delta}\right) e^{-\left(\vartheta u-\frac{1}{\delta}\right) s} \frac{\ln u}{C_{w}\left(\vartheta u-\frac{1}{\delta}\right)} \mathrm{d} u \mathrm{~d} s\right)
$$

so, the Poisson rate is $\frac{a \vartheta \varrho}{\delta} C_{w}$, and jump-sizes $\left\{S_{j}\right\}_{j=1,2, \ldots}$ follow an exponential distribution with rate $\left(\vartheta W-\frac{1}{\delta}\right)$. Here, $W$ is a well-defined random variable with density (B.1), which can be exactly simulated via the A/R scheme of Algorithm B.1. For more details on designing simulation algorithms based on A/R mechanism, see Glasserman (2003) and Asmussen and Glynn (2007).

Algorithm 4.2 (Exact Simulation of Pre-jump Intensity Level for TS-Contagion). For the TScontagion, conditional on the intensity level $\lambda_{T_{i}}$ and the realisation of the $(i+1)^{\text {th }}$ interarrival time $\tau_{i+1}=\tau$, the distribution of the $(i+1)^{\text {th }}$ pre-jump intensity level $\lambda_{T_{i}+\tau^{-}}$can be exactly decomposed by

$$
\lambda_{T_{i}+\tau^{-}} \mid \lambda_{T_{i}} \stackrel{\mathcal{D}}{=} w \lambda_{T_{i}}+\widetilde{T S}+\widetilde{B} \times S+\sum_{k=1}^{\widetilde{N}} S_{k},
$$

where $\widetilde{T S}, \widetilde{B}, S, \widetilde{N}$ and $\left\{S_{k}\right\}_{k=1,2, \ldots}$ are all independent of each other,

- $\widetilde{T S}$ is an TS random variable of

$$
\widetilde{T S} \sim T S\left(\alpha, \frac{\kappa}{w}-\frac{1}{\delta}, \frac{\theta \varrho}{\alpha \delta}\left(1-w^{\alpha}\right)\right), \quad \kappa:=\beta+\frac{1}{\delta}
$$

- $\widetilde{B}$ is a Bernoulli random variable taking 0 with probability $p_{1}$ and 1 with probability $p_{2}$, and

$$
p_{1}:=\frac{w \lambda_{T_{i}}}{\frac{\theta \varrho D}{\delta} \Gamma(1-\alpha)+w \lambda_{T_{i}}}, \quad p_{2}:=\frac{\frac{\theta \varrho D}{\delta} \Gamma(1-\alpha)}{\frac{\theta \varrho D}{\delta} \Gamma(1-\alpha)+w \lambda_{T_{i}}}, \quad D:=\frac{\delta}{\alpha}\left[\left(\kappa-\frac{w}{\delta}\right)^{\alpha}-\beta^{\alpha}\right]
$$


- $S$ is a mixture-gamma random variable of $S \sim \operatorname{Gamma}\left(1-\alpha, \kappa V_{0}-\frac{1}{\delta}\right)$ and

$$
V_{0} \stackrel{\mathcal{D}}{=}\left[\delta \beta+1-\delta\left(\frac{\alpha D}{\delta} U_{2}+\beta^{\alpha}\right)^{\frac{1}{\alpha}}\right]^{-1}, \quad U_{2} \sim U[0,1]
$$

- $\widetilde{N}$ is a Poisson random variable of rate $\frac{\theta \varrho}{\alpha \delta} \kappa \Gamma(1-\alpha) D_{w}$ and

$$
D_{w}:=\int_{1}^{\frac{1}{w}} \frac{1-u^{-\alpha}}{\left(\kappa u-\frac{1}{\delta}\right)^{1-\alpha}} \mathrm{d} u
$$

- $\left\{S_{k}\right\}_{k=1,2, \ldots}$ are i.i.d. with $S_{k} \sim \operatorname{Gamma}\left(1-\alpha, \kappa V-\frac{1}{\delta}\right)$, and $V$ can be exactly simulated via the A/R scheme of Algorithm C.1.

Proof. Algorithm (4.2) is another explicit specification of Theorem 3.2. Similarly as the previous gamma case in Algorithm 4.1, given the Lévy measure (4.5), we can identify that (3.12) is the Laplace transform of $\widetilde{B} \times S$ from the calculation

$$
\begin{aligned}
& \frac{\varrho}{\delta} \int_{0}^{\infty} e^{-v s} \int_{s}^{\frac{s}{w}} e^{\frac{s}{\delta}} e^{-\frac{y}{\delta}} \nu(\mathrm{d} y) \mathrm{d} s+w \lambda_{T_{i}} \\
& \frac{\varrho}{\delta} \int_{0}^{\infty} \int_{s}^{\frac{s}{w}} e^{\frac{s}{\delta}} e^{-\frac{y}{\delta}} \nu(\mathrm{d} y) \mathrm{d} s+w \lambda_{T_{i}} \\
& \frac{\theta \varrho}{\delta} \int_{0}^{\infty} e^{-v s} \int_{1}^{\frac{1}{w}} s^{(1-\alpha)-1} e^{-\left(\kappa u-\frac{1}{\delta}\right) s} u^{-1-\alpha} \mathrm{d} u \mathrm{~d} s+w \lambda_{T_{i}} \\
& =\frac{0}{\int_{0}^{\infty} \int_{1}^{\frac{1}{w}} s^{(1-\alpha)-1} e^{-\left(\kappa u-\frac{1}{\delta}\right) s} u^{-1-\alpha} \mathrm{d} u \mathrm{~d} s+w \lambda_{T_{i}}} \\
& =\frac{w \lambda_{T_{i}}}{\frac{\theta \varrho D}{\delta} \Gamma(1-\alpha)+w \lambda_{T_{i}}} \times 1 \\
& +\frac{\frac{\theta \varrho D}{\delta} \Gamma(1-\alpha)}{\frac{\theta \varrho D}{\delta} \Gamma(1-\alpha)+w \lambda_{T_{i}}} \times \int_{0}^{\infty} e^{-v s} \int_{1}^{\frac{1}{w}} \frac{\left(\kappa u-\frac{1}{\delta}\right)^{1-\alpha}}{\Gamma(1-\alpha)} s^{(1-\alpha)-1} e^{-\left(\kappa u-\frac{1}{\delta}\right) s} \frac{u^{-1-\alpha}}{D\left(\kappa u-\frac{1}{\delta}\right)^{1-\alpha}} \mathrm{d} u \mathrm{~d} s \\
& =p_{1} \times \mathbb{E}\left[e^{-v 0}\right]+p_{2} \times \mathbb{E}\left[e^{-v S}\right] \text {, }
\end{aligned}
$$

where

$$
D=\int_{1}^{\frac{1}{w}} \frac{u^{-1-\alpha}}{\left(\kappa u-\frac{1}{\delta}\right)^{1-\alpha}} \mathrm{d} u=\frac{\delta}{\alpha}\left[\left(\kappa-\frac{w}{\delta}\right)^{\alpha}-\beta^{\alpha}\right] .
$$

So, the outcome of $\widetilde{B} \times S$ is trivially equal to 0 with probability $p_{1}$, or, the random variable $S$ with probability $p_{2}$. $S$ follows a mixture-gamma distribution with the shape parameter $1-\alpha$ and the 
rate parameter $\kappa V_{0}-\frac{1}{\delta}$. Here, $V_{0}$ is a well-defined random variable with density function

$$
f_{V_{0}}(u)=\frac{u^{-\alpha-1}}{D\left(\kappa u-\frac{1}{\delta}\right)^{1-\alpha}}, \quad u \in\left[1, \frac{1}{w}\right]
$$

It can be directly simulated via the explicit inverse transform (4.16), as its CDF is

$$
F_{V_{0}}(u)=\frac{\delta}{\alpha D}\left[u^{-\alpha}\left(\kappa u-\frac{1}{\delta}\right)^{\alpha}-\beta^{\alpha}\right], \quad u \in\left[1, \frac{1}{w}\right]
$$

The first two terms of (3.9), i.e., (3.13), can be expressed by

$$
\begin{aligned}
& \frac{\mathbb{E}\left[e^{-v \lambda_{T_{i}+\tau^{-}}} e^{-\left(\Lambda_{T_{i}+\tau^{-}}-\Lambda_{T_{i}}\right)} \mid \lambda_{T_{i}}\right]}{\mathbb{E}\left[e^{-\left(\Lambda_{T_{i}+\tau^{-}}-\Lambda_{T_{i}}\right)} \mid \lambda_{T_{i}}\right]} \\
= & e^{-v w \lambda_{T_{i}}} \times \exp \left(-\frac{\theta \varrho}{\alpha \delta}\left(1-w^{\alpha}\right) \int_{0}^{\infty}\left(1-e^{-v s}\right) \frac{1}{s^{\alpha+1}} e^{-\left(\frac{\kappa}{w}-\frac{1}{\delta}\right) s} \mathrm{~d} s\right) \\
& \times \exp \left(-\frac{\theta \varrho}{\alpha \delta} \kappa \Gamma(1-\alpha) \int_{0}^{\infty}\left(1-e^{-v s}\right) \int_{1}^{\frac{1}{w}} \frac{\left(\kappa u-\frac{1}{\delta}\right)^{1-\alpha}}{\Gamma(1-\alpha)} s^{(1-\alpha)-1} \frac{1-u^{-\alpha}}{\left(\kappa u-\frac{1}{\delta}\right)^{1-\alpha}} \mathrm{d} u \mathrm{~d} s\right),
\end{aligned}
$$

since the Lévy measure $\nu$ for the TS subordinator is specified in (4.5), and the Laplace exponent of (3.13) can be rewritten by

$$
\begin{aligned}
& \varrho \int_{v}^{\frac{1}{\delta}+\left(v-\frac{1}{\delta}\right) w} \frac{\Phi\left(u-\frac{u-\frac{1}{\delta}}{v-\frac{1}{\delta}} v\right)-\Phi(u)}{1-\delta u} \mathrm{~d} u \\
= & \frac{\varrho}{\delta} \int_{0}^{\infty}\left(1-e^{-v s}\right) \frac{1}{s} e^{\frac{s}{\delta}} \int_{s}^{\frac{s}{w}} \frac{\theta}{y^{\alpha+1}} e^{-\kappa \frac{s}{w}} \mathrm{~d} y \mathrm{~d} s \\
& +\frac{\varrho}{\delta} \int_{0}^{\infty}\left(1-e^{-v s}\right) \frac{e^{\frac{s}{\delta}}}{s} \int_{s}^{\frac{s}{w}} \frac{\theta\left(e^{-\kappa y}-e^{-\frac{\kappa s}{w}}\right)}{y^{\alpha+1}} \mathrm{~d} y \mathrm{~d} s \\
= & \int_{0}^{\infty}\left(1-e^{-v s}\right) \frac{\theta \varrho}{\alpha \delta}\left(1-w^{\alpha}\right) \frac{1}{s^{\alpha+1}} e^{-\left(\frac{\kappa}{w}-\frac{1}{\delta}\right) s} \mathrm{~d} s \\
& +\frac{\theta \varrho}{\alpha \delta} \kappa \Gamma(1-\alpha) \int_{0}^{\infty}\left(1-e^{-v s}\right) \int_{1}^{\frac{1}{w}} \frac{\left(\kappa u-\frac{1}{\delta}\right)^{1-\alpha}}{\Gamma(1-\alpha)} s^{(1-\alpha)-1} e^{-\left(\kappa u-\frac{1}{\delta}\right) s} \frac{1-u^{-\alpha}}{\left(\kappa u-\frac{1}{\delta}\right)^{1-\alpha}} \mathrm{d} u \mathrm{~d} s .
\end{aligned}
$$

So, for (3.13), it consist three components: one deterministic trend, a TS process and a compound Poisson process. In particular, the rate of the compound Poisson process is $\widetilde{N}$ is $\frac{\theta \varrho}{\alpha \delta} \kappa \Gamma(1-\alpha) D_{w}$, and the jump sizes follow a mixture-gamma distribution with the shape parameter $(1-\alpha)$ and the 
rate parameter $\kappa V-\frac{1}{\delta}$. Here, $V$ is a well-defined random variable with density

$$
f_{V}(u)=\frac{1-u^{-\alpha}}{D_{w}\left(\kappa u-\frac{1}{\delta}\right)^{1-\alpha}}, \quad u \in\left[1, \frac{1}{w}\right] .
$$

Overall, we have the conditional Laplace transform of pre-jump intensity level explicitly as

$$
\begin{aligned}
& \mathbb{E}\left[e^{-v \lambda_{T_{i}+\tau_{i+1}^{-}}} \mid \tau_{i+1}=\tau, \lambda_{T_{i}}\right] \\
&= e^{-v w \lambda_{T_{i}}} \times \exp \left(-\frac{\theta \varrho}{\alpha \delta}\left(1-w^{\alpha}\right) \int_{0}^{\infty}\left(1-e^{-v s}\right) \frac{1}{s^{\alpha+1}} e^{-\left(\frac{\kappa}{w}-\frac{1}{\delta}\right) s} \mathrm{~d} s\right) \\
& \times \exp \left(-\frac{\theta \varrho}{\alpha \delta} \kappa \Gamma(1-\alpha) D_{w} \int_{0}^{\infty}\left(1-e^{-v s}\right) \int_{1}^{\frac{1}{w}} \frac{\left(\kappa u-\frac{1}{\delta}\right)^{1-\alpha}}{\Gamma(1-\alpha)} s^{(1-\alpha)-1} \frac{1-u^{-\alpha}}{D_{w}\left(\kappa u-\frac{1}{\delta}\right)^{1-\alpha}} \mathrm{d} u \mathrm{~d} s\right) \\
& \times\left[\frac{w \lambda_{T_{i}}}{\frac{\theta \varrho D}{\delta} \Gamma(1-\alpha)+w \lambda_{T_{i}}}+\frac{\frac{\theta \varrho D}{\delta} \Gamma(1-\alpha)}{\frac{\theta \varrho D}{\delta} \Gamma(1-\alpha)+w \lambda_{T_{i}}} \times u^{-1-\alpha}\right. \\
&\left.\int_{0}^{\infty} e^{-v s} \int_{1}^{\frac{1}{w}} \frac{\left(\kappa u-\frac{1}{\delta}\right)^{1-\alpha}}{\Gamma(1-\alpha)} s^{(1-\alpha)-1} e^{-\left(\kappa u-\frac{1}{\delta}\right) s} \frac{u^{-\alpha}}{D\left(\kappa u-\frac{1}{\delta}\right)^{1-\alpha}} \mathrm{d} u \mathrm{~d} s\right] .
\end{aligned}
$$

We can identify from the Laplace transforms above that, the distribution of the $(i+1)^{\text {th }}$ pre-jump intensity level $\lambda_{T_{i}+\tau^{-}}$conditional on $\lambda_{T_{i}}$ is exactly equal in distribution to the sum of four simple elements provided in (4.14). All these components can be simulated exactly. To simulate the TS random variable, one could use existing algorithms provided in Brix (1999), Devroye (2009), Hofert (2011) or Backward Recursive (BR) Scheme provided in Dassios et al. (2018) . And to sample the compound Poisson random variable $\widetilde{N}$, one first needs to generate the intermediate random variable $V$ with density (4.19). Since there is no closed form for the inverse function of the CDF of $V$, we have to rely on the A/R scheme of Algorithm C.1.

Exact Simulation of Self-exciting Jumps Conditional on the realisations of the interarrival time and pre-jump intensity level as above, the associated self-exciting jump can be easily simulated by just following Algorithm 3.3 in general both for gamma and TS contagion processes.

\section{Numerical Experiments}

In this section, let us take the TS-contagion model as an example, to illustrate the performance of our exact scheme through extensive numerical experiments, and postpone the implementation for the gamma-contagion model later in Section 6 with more financial applications. The simulation experiments here and in the other parts of this paper are all conducted on a desktop PC with an 
Intel Core i7-3770S CPU@3.10GHz processor, 8.00GB RAM, Windows 7, 64-bit Operating System; the algorithms are coded and performed in MatLab (R2014a), and the computation time is measured by the elapsed CPU time in seconds. The true value of the conditional expectation of $N_{T}$ for any fixed time $T>0$ provided in Proposition 2.1 is used to numerically validate and test our algorithms. The associated errors from the true values are reported by three standard measures:

1. Error $=$ estimated value - true value;

2. Relative error (error \%) $=\frac{\text { estimated value }- \text { true value }}{\text { true value }}$

3. Root mean square error $\mathrm{RMSE}=\sqrt{\mathrm{bias}^{2}+\mathrm{SE}^{2}}$, where the $\mathrm{SE}$ is the standard error of simulation output, and the bias is the difference between the expectation of the estimator and the associated true (theoretical) value; for the algorithm of exact simulation here, the bias is conventionally set to zero.

We implement Algorithm 3.3 for the TS and IG cases in a fixed period of $[0, T]$ with and without self-exciting jumps:

Case I: Jump process with non-Gaussian OU intensity (of Definition 2.1);

Case II: Self-exciting jump process with non-Gaussian OU intensity (of Definition 2.2).

Note that, as an intermediate step, we have to generate the random variable $\widetilde{T S}$ of (4.15) in Algorithm 4.2 for the general TS case. There are several algorithms available in the literature, including approximation-based algorithms such as infinite series representation (Rosiński, 2001), and exact algorithms such as simple stable rejection (SSR) (Brix, 1999), double rejection algorithm (Devroye, 2009), fast rejection algorithm (Hofert, 2011) and backward recursive (BR) scheme (Dassios et al., 2018). However, the choice of algorithms is indeed not our focus of this paper. Just for the propose of demonstration here, we adopt the BR scheme. It works extremely efficiently for some families including the one with stability index of binary fractions $\frac{1}{2^{n}}, n=1,2, \ldots$, which can be easily simulated by recursively generating IG random variables without $A / R$ mechanism. For a general parameter setting, one could simply adopt other algorithms such as the SSR scheme of Algorithm E.1.

For numerical implementation, we further assume that, the sizes of self-exciting jumps follow an exponential distribution of rate $\gamma>0$, i.e. $X_{i} \sim \operatorname{Exp}(\gamma)$, and the stable index takes the values of, say, $\alpha=1 / 4$ for the TS case and $\alpha=1 / 2$ for the IG case, respectively. $\widetilde{T S}$ of (4.15) is simulated using the BR scheme particularly designed for $\alpha=1 / 4$ in Algorithm D.1, and the parameters are set by: 
1. TS Case I: $\left(\delta, \varrho ; \alpha, \beta, \theta ; \lambda_{0}\right)=(1.0,0.5 ; 0.25,0.2,0.25 ; 0.5)$;

2. TS Case II: $\left(\delta, \varrho ; \alpha, \beta, \theta ; \gamma ; \lambda_{0}\right)=(1.0,0.5 ; 0.25,0.2,0.25 ; 5.0 ; 0.5)$;

3. IG Case I: $\left(\delta, \varrho ; c ; \lambda_{0}\right)=(1.0,0.5 ; 0.5 ; 0.5)$;

4. IG Case II: $\left(\delta, \varrho ; c ; \gamma ; \lambda_{0}\right)=(1.0,0.5 ; 0.5 ; 4.0 ; 0.5)$.

Simulated sample paths of the point processes within a long period of $t \in[0,500]$ with the associated histograms are plotted in Figure 3, where the clustering or "contagious" arrivals of jumps can be clearly presented. Furthermore, to measure the accuracy and efficiency of our scheme, we carry out the convergence analysis: Figure 4 presents $\log -\log$ plots for the RMSE against the CPU time for each case in two different time horizons $T=2,5$, respectively, and the associated results in detail are reported in Table 3. Overall, from these numerical results reported in this section, it is evident that our exact scheme can achieve a very high level of accuracy and efficiency.

To be even more prudent, the simulation for the interarrival time based on the simplified thinning scheme of Algorithm 3.2, as an intermediate step, can be also tested separately. To numerically assess its accuracy and efficiency, we compare the simulated results of $V^{*}$ with its theoretical tail distribution $\operatorname{Pr}\left\{V^{*}>\tau\right\}$ as specified in (3.7), which can be calculated explicitly by substituting the Laplace exponent $\Phi$ from (4.6). We set the parameters by $(\delta, \varrho ; \alpha, \beta, \theta)=(0.5,1.0 ; 0.9,0.2,0.25)$, and each estimation is based on $10^{5}$ replications. Error percentages (Error\%) for measuring relative errors are reported in Table 2. The total CPU time for producing the whole Table 2 is only 12.64 seconds, and the error percentages are all very tiny.

\section{Comprehensive Risk Analysis for A Large Portfolio Facing Conta- gious Losses and Unexpected Exogenous Gamma Shocks}

It has now been widely recognised among academics and financial practitioners that, risk spreads through highly interconnected business networks, and defaults could trigger more defaults through a "domino" effect. The resulting losses presented in financial markets could be amplified. As earlier mentioned in Section 4, gamma distribution is a poplar building block in financial applications. Especially, it plays an important role in credit risk modelling. For instance, both the widely-used framework of CreditRisk ${ }^{+}$(1997) in the banking industry and influential papers by Gordy (2000, 2003) and Elsinger et al. (2006) in the literature, assumed that, macroeconomic factors are driven by independent gamma-distributed random variables ${ }^{9}$. More recent evidences have been found by

\footnotetext{
${ }^{9}$ Besides, it can be also equipped as a fundamental risk driver for price movements, e.g. the popular variance Gamma model (Madan and Seneta, 1990; Madan et al., 1998).
} 



Figure 3: Simulated sample paths of the point processes and the associated time-series plots for Case I\&II: TS Case I $\left(\delta, \varrho ; \alpha, \beta, \theta ; \lambda_{0}\right)=(1.0,0.5 ; 0.25,0.2,0.25 ; 0.5)$, TS Case II $\left(\delta, \varrho ; \alpha, \beta, \theta ; \gamma ; \lambda_{0}\right)=$ $(1.0,0.5 ; 0.25,0.2,0.25 ; 5.0 ; 0.5)$; IG Case I $\left(\delta, \varrho ; c ; \lambda_{0}\right)=(1.0,0.5 ; 0.5 ; 0.5)$, IG Case II $\left(\delta, \varrho ; c ; \gamma ; \lambda_{0}\right)=(1.0,0.5 ; 0.5 ; 4.0 ; 0.5)$ 
Table 1: Simulation results for Case I\&II: TS Case I $\left(\delta, \varrho ; \alpha, \beta, \theta ; \lambda_{0}\right)=(1.0,0.5 ; 0.25,0.2,0.25 ; 0.5)$, TS Case II $\left(\delta, \varrho ; \alpha, \beta, \theta ; \gamma ; \lambda_{0}\right)=(1.0,0.5 ; 0.25,0.2,0.25 ; 5.0 ; 0.5)$; IG Case I $\left(\delta, \varrho ; c ; \lambda_{0}\right)=$ $(1.0,0.5 ; 0.5 ; 0.5)$, IG Case II $\left(\delta, \varrho ; c ; \gamma ; \lambda_{0}\right)=(1.0,0.5 ; 0.5 ; 4.0 ; 0.5)$

\begin{tabular}{|c|c|c|c|c|c|c|c|}
\hline Case & Paths & True & Simulation & Error & Error\% & RMSE & CPU Time (sec) \\
\hline \multicolumn{8}{|c|}{ TS Case } \\
\hline \multirow[t]{7}{*}{ Case $\mathbf{I}, T=2$} & 100 & 1.0138 & 0.9300 & -0.0838 & $-8.2683 \%$ & 0.1281 & 0.11 \\
\hline & 400 & 1.0138 & 1.0525 & 0.0387 & $3.8146 \%$ & 0.0834 & 0.36 \\
\hline & 1,600 & 1.0138 & 1.0363 & 0.0224 & $2.2118 \%$ & 0.0398 & 1.37 \\
\hline & 6,400 & 1.0138 & 1.0184 & 0.0046 & $0.4548 \%$ & 0.0208 & 5.29 \\
\hline & 25,600 & 1.0138 & 1.0167 & 0.0029 & $0.2853 \%$ & 0.0099 & 20.53 \\
\hline & 102,400 & 1.0138 & 1.0133 & -0.0005 & $-0.0509 \%$ & 0.0049 & 81.74 \\
\hline & 409,600 & 1.0138 & 1.0127 & -0.0012 & $-0.1147 \%$ & 0.0024 & 321.78 \\
\hline \multirow[t]{7}{*}{ Case II, $T=5$} & 100 & 2.5488 & 2.5500 & 0.0012 & $0.0472 \%$ & 0.2879 & 0.22 \\
\hline & 400 & 2.5488 & 2.4700 & -0.0788 & $-3.0915 \%$ & 0.1466 & 0.73 \\
\hline & 1,600 & 2.5488 & 2.6413 & 0.0925 & $3.6274 \%$ & 0.0815 & 2.92 \\
\hline & 6,400 & 2.5488 & 2.5308 & -0.0180 & $-0.7068 \%$ & 0.0380 & 11.08 \\
\hline & 25,600 & 2.5488 & 2.5523 & 0.0035 & $0.1361 \%$ & 0.0190 & 44.29 \\
\hline & 102,400 & 2.5488 & 2.5589 & 0.0101 & $0.3948 \%$ & 0.0095 & 177.92 \\
\hline & 409,600 & 2.5488 & 2.5437 & -0.0051 & $-0.1998 \%$ & 0.0048 & 706.82 \\
\hline \multirow{7}{*}{ Case II, $T=2$} & 100 & 1.1406 & 1.1500 & 0.0094 & $0.8281 \%$ & 0.1520 & 0.14 \\
\hline & 400 & 1.1406 & 1.0925 & -0.0481 & $-4.2133 \%$ & 0.0909 & 0.36 \\
\hline & 1,600 & 1.1406 & 1.1581 & 0.0176 & $1.5404 \%$ & 0.0453 & 1.48 \\
\hline & 6,400 & 1.1406 & 1.1233 & -0.0173 & $-1.5145 \%$ & 0.0228 & 5.71 \\
\hline & 25,600 & 1.1406 & 1.1468 & 0.0062 & $0.5472 \%$ & 0.0114 & 23.21 \\
\hline & 102,400 & 1.1406 & 1.1305 & -0.0101 & $-0.8835 \%$ & 0.0056 & 90.92 \\
\hline & 409,600 & 1.1406 & 1.1454 & 0.0049 & $0.4282 \%$ & 0.0028 & 365.82 \\
\hline \multirow[t]{8}{*}{ Case II, $T=5$} & 100 & 3.0290 & 2.9900 & -0.0390 & $-1.2891 \%$ & 0.3932 & 0.23 \\
\hline & 400 & 3.0290 & 3.0875 & 0.0585 & $1.9298 \%$ & 0.1903 & 0.87 \\
\hline & 1,600 & 3.0290 & 3.0413 & 0.0122 & $0.4029 \%$ & 0.0923 & 3.34 \\
\hline & 6,400 & 3.0290 & 3.0961 & 0.0670 & $2.2135 \%$ & 0.0491 & 13.54 \\
\hline & 25,600 & 3.0290 & 3.0095 & -0.0196 & $-0.6456 \%$ & 0.0234 & 52.28 \\
\hline & 102,400 & 3.0290 & 3.0264 & -0.0026 & $-0.0862 \%$ & 0.0117 & 207.73 \\
\hline & 409,600 & 3.0290 & 3.0308 & 0.0017 & $0.0569 \%$ & 0.0058 & 831.64 \\
\hline & \multicolumn{7}{|c|}{ IG Case } \\
\hline \multirow[t]{7}{*}{ Case $\mathbf{I}, T=2$} & 100 & 1.5677 & 1.5000 & -0.0677 & $-4.3165 \%$ & 0.1673 & 0.06 \\
\hline & 400 & 1.5677 & 1.6025 & 0.0348 & $2.2219 \%$ & 0.0948 & 0.19 \\
\hline & 1,600 & 1.5677 & 1.6431 & 0.0755 & $4.8134 \%$ & 0.0573 & 0.56 \\
\hline & 6,400 & 1.5677 & 1.5311 & -0.0366 & $-2.3330 \%$ & 0.0257 & 2.17 \\
\hline & 25,600 & 1.5677 & 1.5745 & 0.0068 & $0.4328 \%$ & 0.0134 & 8.50 \\
\hline & 102,400 & 1.5677 & 1.5743 & 0.0066 & $0.4229 \%$ & 0.0068 & 33.77 \\
\hline & 409,600 & 1.5677 & 1.5687 & 0.0011 & $0.0673 \%$ & 0.0033 & 135.05 \\
\hline \multirow[t]{7}{*}{ Case $\mathbf{I}, T=5$} & 100 & 4.5034 & 4.1500 & -0.3534 & $-7.8468 \%$ & 0.3880 & 0.11 \\
\hline & 400 & 4.5034 & 4.3075 & -0.1959 & $-4.3494 \%$ & 0.2118 & 0.27 \\
\hline & 1,600 & 4.5034 & 4.3394 & -0.1640 & $-3.6416 \%$ & 0.1026 & 1.06 \\
\hline & 6,400 & 4.5034 & 4.5084 & 0.0051 & $0.1125 \%$ & 0.0546 & 4.23 \\
\hline & 25,600 & 4.5034 & 4.5268 & 0.0234 & $0.5202 \%$ & 0.0272 & 16.83 \\
\hline & 102,400 & 4.5034 & 4.5236 & 0.0203 & $0.4500 \%$ & 0.0135 & 67.08 \\
\hline & 409,600 & 4.5034 & 4.5037 & 0.0003 & $0.0067 \%$ & 0.0068 & 268.24 \\
\hline \multirow[t]{7}{*}{ Case II, $T=2$} & 100 & 1.8035 & 1.7100 & -0.0935 & $-5.1832 \%$ & 0.21 & 0.11 \\
\hline & 400 & 1.8035 & 1.7925 & -0.0110 & $-0.6087 \%$ & 0.13 & 0.16 \\
\hline & 1,600 & 1.8035 & 1.7644 & -0.0391 & $-2.1682 \%$ & 0.06 & 0.58 \\
\hline & 6,400 & 1.8035 & 1.7758 & -0.0277 & $-1.5357 \%$ & 0.03 & 2.31 \\
\hline & 25,600 & 1.8035 & 1.8043 & 0.0008 & $0.0454 \%$ & 0.02 & 9.31 \\
\hline & 102,400 & 1.8035 & 1.8025 & -0.0010 & $-0.0569 \%$ & 0.01 & 36.07 \\
\hline & 409,600 & 1.8035 & 1.8039 & 0.0004 & $0.0232 \%$ & 0.00 & 145.36 \\
\hline \multirow[t]{7}{*}{ Case II, $T=5$} & 100 & 5.5817 & 5.2400 & -0.3417 & $-6.1216 \%$ & 0.4584 & 0.11 \\
\hline & 400 & 5.5817 & 5.4000 & -0.1817 & $-3.2550 \%$ & 0.2753 & 0.34 \\
\hline & 1,600 & 5.5817 & 5.6300 & 0.0483 & $0.8656 \%$ & 0.1344 & 1.25 \\
\hline & 6,400 & 5.5817 & 5.5902 & 0.0085 & $0.1517 \%$ & 0.0698 & 4.99 \\
\hline & 25,600 & 5.5817 & 5.5648 & -0.0169 & $-0.3024 \%$ & 0.0347 & 19.83 \\
\hline & 102,400 & 5.5817 & 5.5777 & -0.0040 & $-0.0713 \%$ & 0.0173 & 77.70 \\
\hline & 409,600 & 5.5817 & 5.5738 & -0.0079 & $-0.1419 \%$ & 0.0087 & 315.71 \\
\hline
\end{tabular}



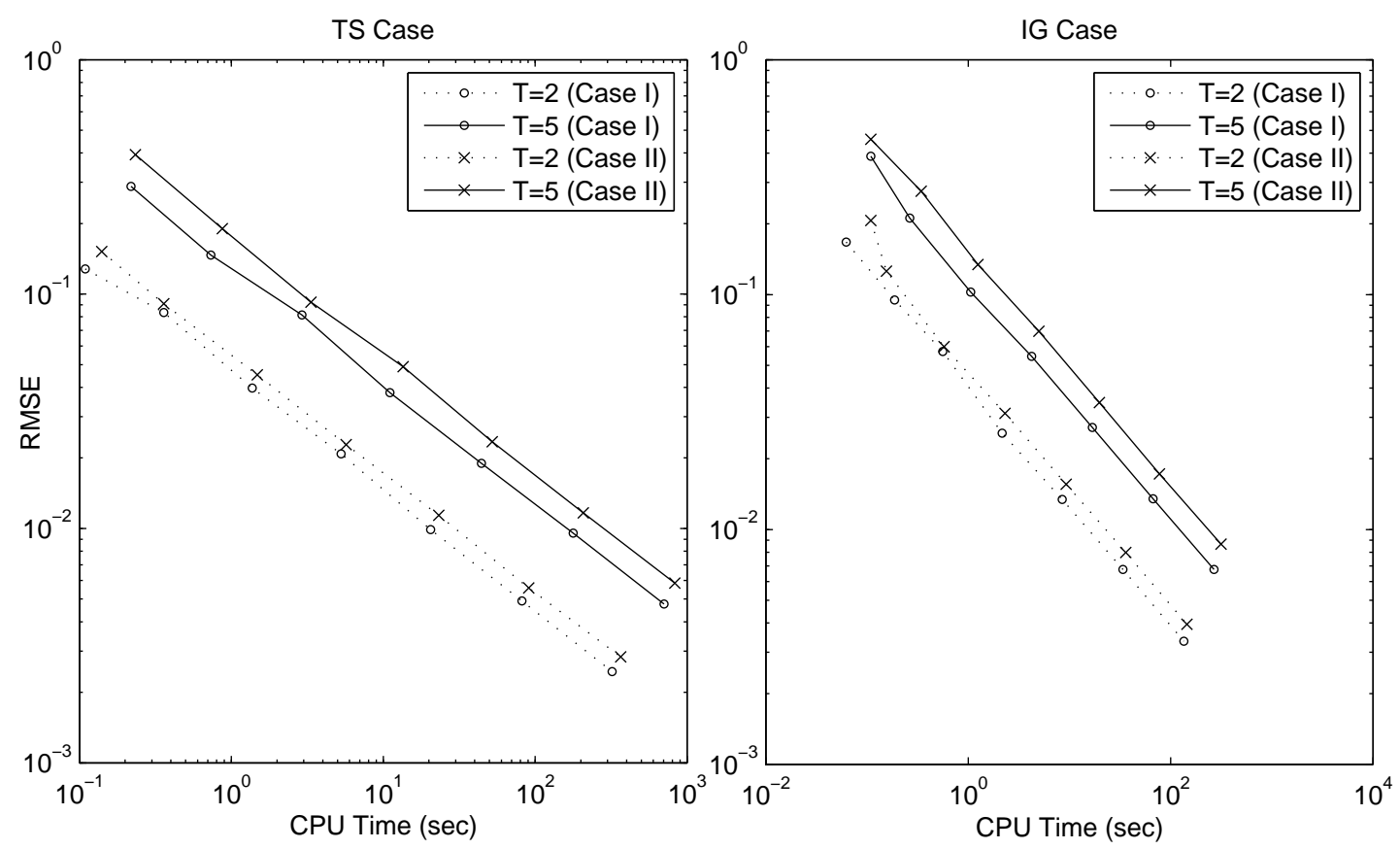

Figure 4: Convergence analysis via RMSE v.s. CPU time by log-log plots for Case I\&II: TS Case $\mathrm{I}\left(\delta, \varrho ; \alpha, \beta, \theta ; \lambda_{0}\right)=(1.0,0.5 ; 0.25,0.2,0.25 ; 0.5)$, TS Case II $\left(\delta, \varrho ; \alpha, \beta, \theta ; \gamma ; \lambda_{0}\right)=$ $(1.0,0.5 ; 0.25,0.2,0.25 ; 5.0 ; 0.5)$; IG Case I $\left(\delta, \varrho ; c ; \lambda_{0}\right)=(1.0,0.5 ; 0.5 ; 0.5)$, IG Case II $\left(\delta, \varrho ; c ; \gamma ; \lambda_{0}\right)=(1.0,0.5 ; 0.5 ; 4.0 ; 0.5)$

Table 2: Comparison between the theoretical formulas and the associated simulation results for the simplified thinning scheme of Algorithm 3.2 with each estimation based on $10^{5}$ replications

\begin{tabular}{c|ccc}
\hline$\tau$ & $\operatorname{Pr}\left\{V^{*}>\tau\right\}$ & Simulation & Error $\%$ \\
\hline 0.1 & $98.66 \%$ & $98.62 \%$ & $-0.0367 \%$ \\
0.2 & $94.87 \%$ & $94.80 \%$ & $-0.0752 \%$ \\
0.3 & $89.10 \%$ & $89.01 \%$ & $-0.0965 \%$ \\
0.4 & $81.85 \%$ & $81.81 \%$ & $-0.0499 \%$ \\
0.5 & $73.66 \%$ & $73.60 \%$ & $-0.0819 \%$ \\
0.6 & $65.01 \%$ & $64.86 \%$ & $-0.2268 \%$ \\
0.7 & $56.35 \%$ & $56.12 \%$ & $-0.3943 \%$ \\
0.8 & $48.00 \%$ & $47.89 \%$ & $-0.2333 \%$ \\
0.9 & $40.24 \%$ & $40.15 \%$ & $-0.2239 \%$ \\
1.0 & $33.22 \%$ & $33.13 \%$ & $-0.2773 \%$ \\
\hline
\end{tabular}

Giesecke et al. (2011b) that, long periods with relatively few defaults follow by episodes of significant clustering of defaults and the resulting distribution of default rates is highly skewed towards large values. This motivates us to adopt our new model of gamma contagion as an example for applications in risk management for a portfolio facing "domino" effect of losses. We assume that exogenous commonly-shared risk is dynamically powered by a gamma process. More precisely, we adopt the gamma distribution as the fundamental driver of randomness (or gamma shock) to construct the $O U-\Gamma$ interarrival intensity for a point process $N_{t}$ in Definition 2.2. A simulated path of this interarrival intensity process within the time period $t \in[0,5]$ based on the parameter setting $\left(\delta, \varrho ; a, b ; \lambda_{0}\right)=(1.0,1.0 ; 4.0,0.5 ; 2.0)$ is earlier plotted in Figure 1 , where we can observe relatively high-frequent and small-size shocks. In fact, it is named as the $O U-\Gamma$ process by 
Barndorff-Nielsen and Shephard (2003a), which has become a very popular tool for modelling stochastic volatilities in a continuous-time setup. Hainaut and Devolder (2008) used it as a special case of Cox processes to model human-mortality rates, and applied to actuarial valuation in insurance. Eberlein et al. (2013) treated it as a one-factor model for describing the evolution of instantaneous interest rates.

In reality, contagion may be triggered by losses or defaults of banks or other financial institutions through inter-institutional lendings in the interbank market, or, it may be further amplified due to some common asset holdings of overlapping portfolios (Caccioli et al., 2014). We offer some numerical examples of comprehensive risk analysis for a large portfolio facing contagious defaults and losses. We construct a simple contagious loss process to capture the propagated defaults for a generic large pool of financial institutions (banks for short) ${ }^{10}$ within a financial system. The aggregate loss process of this large portfolio by time $t$ is

$$
L_{t}=\sum_{i=1}^{N_{t}} L_{i}, \quad t \geq 0,
$$

where $N_{t}$ is a $\Gamma$-contagion process, and $L_{i} \geq 0$ is the absolute value of the loss size for the $i^{\text {th }}$ default, of which the mean is denoted by $\mu_{L}:=\mathbb{E}\left[L_{i}\right]$ for any index $i$. We assume that the sizes of self-exciting jumps in (2.3) generally satisfy

$$
X_{i}=\varpi_{i} \times g\left(H_{i}\right),
$$

where

- $H_{i}$ is the history of the loss path until time $t$, i.e. $H_{i}:=\left\{L_{j}\right\}_{j<i} \cup\left\{T_{j}\right\}_{j \leq i}$;

- $\varpi_{i}>0$ is the amplification multiplier ${ }^{11}$, which might be dependent on the degree of financial connectivity of the underlying company $i$ to others, or, the effects of policymakers' interventions to limit the extent of contagion;

- $g(\cdot)$ is a general non-negative function ${ }^{12}$ of the losses.

\footnotetext{
${ }^{10}$ The following framework of course would generically work for other types of similar institutions.

${ }^{11}$ The amplification mechanisms during financial crises in detail were described and analysed in Brunnermeier (2009); Brunnermeier and Pedersen (2009) and more recently in ?.

${ }^{12}$ Since the jump sizes in (6.1) follow a $\mathcal{F}_{T_{i}^{-}}$-measurable distribution as explained earlier in Definition 2.2, similarly, the function $g(\cdot)$ is also very general, and it can include all past losses, i.e.,

$$
g(\cdot)=g\left(T_{1}, T_{2}, \ldots, T_{i}, L_{1}, \ldots, L_{i-1}\right),
$$

which could adopt functional forms, for example, assigning weight to each loss size according to its loss time or similarly as autoregressive time series models.
} 
In fact, (6.1) provides a channel for contagion (or feedback) effects of market participants' reactions to adverse scenarios. The economic interpretation for this model is that, the impacts and the timing of unexpected exogenous gamma shocks acting on the entire portfolio as macroeconomic scenarios are modelled by a mean-reverting gamma-driven OU process. Each of the shocks may not lead to an immediate default but acts on the underlying intensity via a positive jump, which increases the default probability afterwards. Meanwhile, endogenic shocks, i.e. contagious losses due to the propagated defaults, are modelled by self-exciting jumps, and the associated magnitudes can be captured by jump sizes $\left\{X_{i}\right\}_{i=1,2, \ldots}$.

The great flexility of our exact simulation scheme allows us to accurately and efficiently generate highly comprehensive scenarios for risk assessment. In general, our algorithms can simulate sample paths when loss sizes $L_{i}$ may depend on the entire history of $N_{t}$ and $\lambda_{t}$ before or at time $T_{i}$. We discuss several circumstances which can be captured by our models as follows.

\subsection{A Simple Benchmark Model}

The loss occurred within a financial institution may spread via various business channels and eventually trigger subsequent losses of others in markets. Intuitively, a larger loss may make a larger impact. For illustration convenience, we assume that the sizes of self-exciting jumps satisfy

$$
X_{i}=\bar{\varpi} \times L_{i}
$$

where $\bar{\varpi}>0$ is the average amplification multiplier, meaning that each investment has a linear and homogenous amplification effect. We further assume that each loss size is exponentially distributed, i.e. $L_{i} \sim \operatorname{Exp}(\ell), \ell>0$ with mean $\mu_{L}:=1 / \ell$. To assess the overall risk of this portfolio, we implement the exact simulation of Algorithm 3.3, 4.1 with (Case I) and without (Case II) contagion in the fixed time period $[0, t]$, respectively:

1. Case I: $\left(\delta, \varrho ; a, b ; \lambda_{0}\right)=(0.5,0.5 ; 0.5,2.0 ; 0.5)$;

2. Case II: $\left(\delta, \varrho ; a, b ; \ell, \bar{\varpi} ; \lambda_{0}\right)=(0.5,0.5 ; 0.5,2.0 ; 8.0,2.0 ; 0.5)$.

We concentrate on the default number $N_{t}$ in the system. Case I or II can be considered as a benchmark model, as by Proposition 2.1 the expected default number has analytical forms:

Proposition 6.1 (Expectation of $N_{t}$ ). The expected default number until time $t$ is given by

$$
\mathbb{E}\left[N_{t} \mid \lambda_{0}\right]=\lambda_{0} \frac{1-e^{-\eta t}}{\eta}+\frac{\varrho}{\eta}\left(t-\frac{1-e^{-\eta t}}{\eta}\right) \frac{a}{b}, \quad \eta \neq 0
$$


where

$$
\eta= \begin{cases}\delta, & \text { for Case I, } \\ \delta-\bar{\varpi} / \ell, & \text { for Case II. }\end{cases}
$$

To explore the models, let us first carry out a sensitivity analysis for the expected default number $\mathbb{E}\left[N_{t} \mid \lambda_{0}\right]$ with respect to (w.r.t.) their key parameters for controlling the external gamma shocks, $a$ and $b$, with and without contagion, and the results are provided respectively in Figure 5. Numerical tests for our algorithms are based on the true means (6.2). The associated errors are reported by three standard measures are reported in Table 3. Convergence analysis via log-log plots of the RMSE against the CPU time for Case I\&II and $t=2,5$ is presented in Figure 6. We can observe that, simulations are pretty fast with very tiny errors, which provides the numerical evidence of accuracy and efficiency for our algorithms.

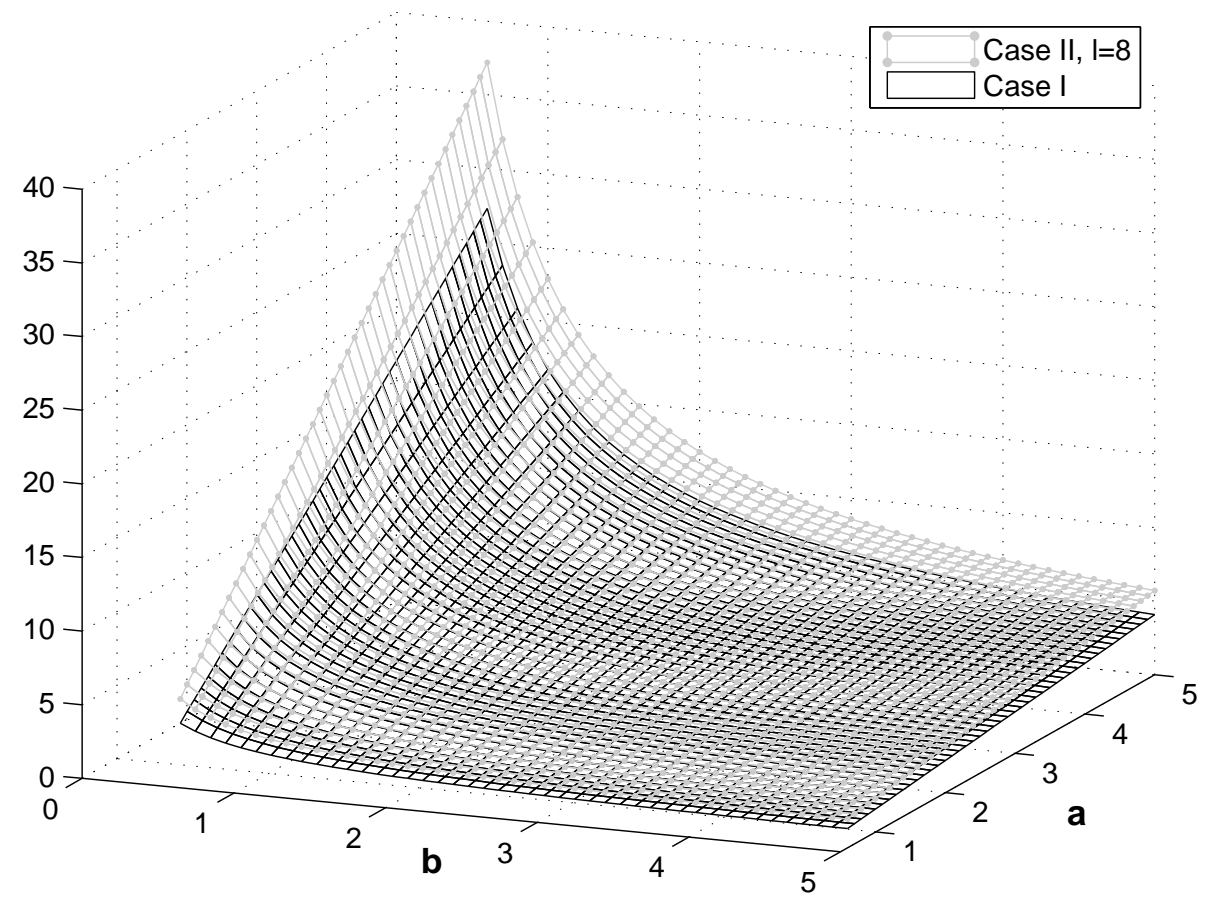

Figure 5: Sensitivity analysis for the expected number of defaults $\mathbb{E}\left[N_{t=5} \mid \lambda_{0}\right]$ w.r.t. $a$ and $b$ based on the parameters $\left(\delta, \varrho ; \bar{\varpi} ; \lambda_{0}\right)=(0.5,0.5 ; 2.0 ; 0.5)$

\subsection{A Model with Contagion Threshold}

In reality, each loss might not necessarily cause a contagion immediately throughout the entire system. Contagion may be only triggered when the loss surpasses a certain high level, i.e. contagion is likely to occur only in severe scenarios, which has also been reported in Elsinger et al. (2006). This circumstance could be modelled by a mixture of Case I and II, by assuming that the sizes of self-exciting jumps $X_{i}$ satisfy

$$
X_{i}=\varpi_{i} \times\left(L_{i}-K_{i}\right)^{+},
$$




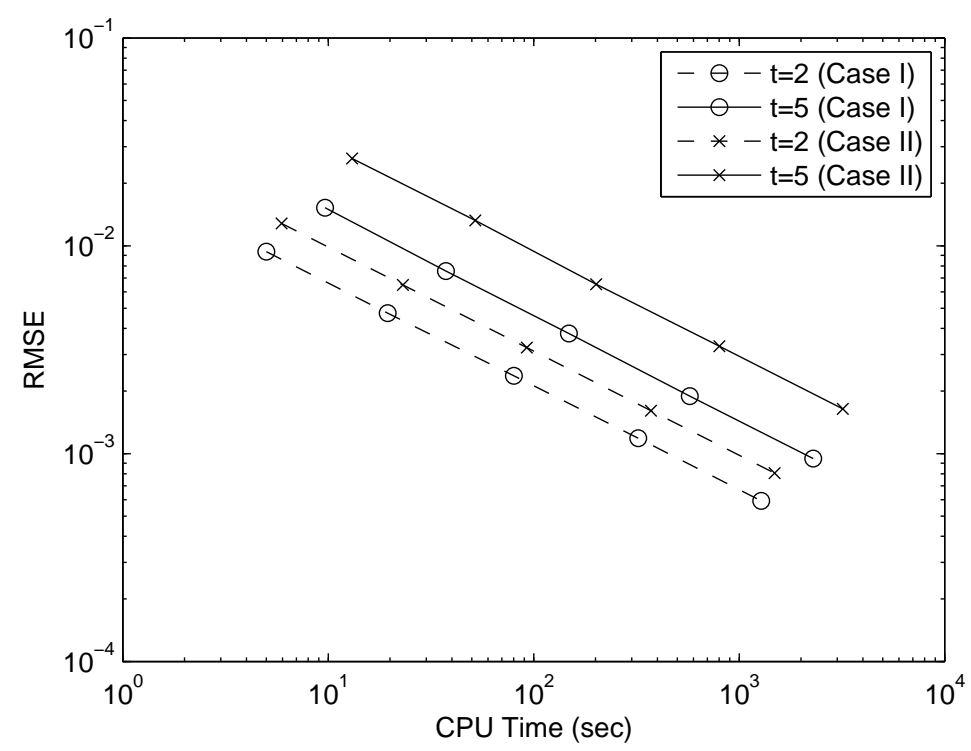

Figure 6: Convergence analysis via the log-log plots of the RMSE v.s. the CPU time for Case I\&II and $t=2,5:\left(\delta, \varrho ; a, b ; \lambda_{0}\right)=(0.5,0.5 ; 0.5,2.0 ; 0.5)$ for Case $I$, and $\left(\delta, \varrho ; a, b ; \ell, \bar{\varpi} ; \lambda_{0}\right)=$ $(0.5,0.5 ; 0.5,2.0 ; 8.0,2.0 ; 0.5)$ for Case II

Table 3: Simulation results for Case I\&II and time $t=2,5:\left(\delta, \varrho ; a, b ; \lambda_{0}\right)=(0.5,0.5 ; 0.5,2.0 ; 0.5)$ for Case I, and $\left(\delta, \varrho ; a, b ; \ell, \bar{\varpi} ; \lambda_{0}\right)=(0.5,0.5 ; 0.5,2.0 ; 8.0,2.0 ; 0.5)$ for Case II

\begin{tabular}{c|c|cccccc}
\hline Case & Paths & True & Simulation & Error & Error\% & RMSE & CPU Time (sec) \\
\hline \hline Case I, $t=2$ & 10,000 & 0.8161 & 0.8154 & -0.0007 & $-0.08 \%$ & 0.0094 & 5.00 \\
& 40,000 & 0.8161 & 0.8151 & -0.0010 & $-0.12 \%$ & 0.0047 & 19.50 \\
& 160,000 & 0.8161 & 0.8135 & -0.0026 & $-0.31 \%$ & 0.0024 & 80.13 \\
& 640,000 & 0.8161 & 0.8159 & -0.0001 & $-0.02 \%$ & 0.0012 & 322.89 \\
& $2,560,000$ & 0.8161 & 0.8163 & 0.0003 & $0.03 \%$ & 0.0006 & $1,280.63$ \\
\hline Case I, $t=5$ & 10,000 & 1.7090 & 1.7297 & 0.0207 & $1.21 \%$ & 0.0152 & 9.66 \\
& 40,000 & 1.7090 & 1.6993 & -0.0097 & $-0.57 \%$ & 0.0076 & 37.45 \\
& 160,000 & 1.7090 & 1.7085 & -0.0004 & $-0.02 \%$ & 0.0038 & 148.38 \\
& 640,000 & 1.7090 & 1.7079 & -0.0010 & $-0.06 \%$ & 0.0019 & 575.55 \\
& $2,560,000$ & 1.7090 & 1.7083 & -0.0006 & $-0.04 \%$ & 0.0009 & $2,291.14$ \\
\hline Case II, $t=2$ & 10,000 & 1.0000 & 0.9983 & -0.0017 & $-0.17 \%$ & 0.0128 & 5.94 \\
& 40,000 & 1.0000 & 1.0054 & 0.0054 & $0.54 \%$ & 0.0065 & 23.13 \\
& 160,000 & 1.0000 & 1.0073 & 0.0073 & $0.73 \%$ & 0.0032 & 92.63 \\
& 640,000 & 1.0000 & 1.0000 & 0.0000 & $0.00 \%$ & 0.0016 & 371.98 \\
& $2,560,000$ & 1.0000 & 0.9995 & -0.0005 & $-0.05 \%$ & 0.0008 & $1,489.06$ \\
\hline Case II, $t=5$ & 10,000 & 2.5000 & 2.5060 & 0.0060 & $0.24 \%$ & 0.0263 & 13.06 \\
& 40,000 & 2.5000 & 2.4964 & -0.0036 & $-0.14 \%$ & 0.0132 & 52.00 \\
& 160,000 & 2.5000 & 2.4882 & -0.0118 & $-0.47 \%$ & 0.0065 & 201.44 \\
& 640,000 & 2.5000 & 2.4972 & -0.0028 & $-0.11 \%$ & 0.0033 & 800.75 \\
& $2,560,000$ & 2.5000 & 2.4983 & -0.0017 & $-0.07 \%$ & 0.0016 & $3,197.61$ \\
\hline
\end{tabular}


Table 4: Quantiles of the default number $N_{t=5}$, estimated from $10^{6}$ replications based on the parameter setting $\left(\delta, \varrho ; a, b ; \ell, \bar{\varpi} ; \lambda_{0}\right)=(0.5,0.5 ; 0.5,2.0 ; 8.0,2.0 ; 0.5)$, with homogenous contagion thresholds $K=\infty, 1 / 8,0$, respectively

\begin{tabular}{|c|c|c|c|c|c|c|c|c|}
\hline$K_{K} \quad$ Quantile & $5 \%$ & $25 \%$ & $50 \%$ & $75 \%$ & $95 \%$ & Mean & Min & Max \\
\hline$\infty$ & 0 & 1 & 1 & 2 & 5 & 1.7075 & 0 & $\overline{17}$ \\
\hline $1 / 8$ & 0 & 1 & 2 & 3 & 5 & 1.9417 & 0 & 22 \\
\hline 0 & 0 & 1 & 2 & 4 & 8 & 2.5021 & 0 & 36 \\
\hline
\end{tabular}

where $K_{i} \geq 0$ is the contagion threshold (i.e. the threshold that triggers the contagion effect of the $i^{\text {th }}$ loss $L_{i}$ ), and the contagion has been partially capped. When a bank is more vulnerable, its threshold is more easy to be reached. Alternatively, we may interpret $K_{i}$ as a capital buffer, and it could be a certain quantile of the loss distribution $L_{i}$. If we assign the same quantile to all banks, it is equivalently meaning that, an identical economic capital applies to all banks, which is the assumption made by Elsinger et al. (2006, p.1306-1308). If the magnitude of loss overshoots the threshold, the bank may become insolvent, and this risk may then spread to other banks (through the interbank market) resulting in a climb in the default intensity of the entire system (but would not cause other banks default immediately). If the thresholds are very high comparing to the levels of losses, then, it corresponds to a "weak contagion" environment; whereas if the thresholds are very low, then, it is for a "strong contagion" environment.

With the contagion threshold, contagion could be partially or fully triggered. Here for numerical illustration, we assume that, losses are exponentially distributed and the amplification multipliers and contagion thresholds are homogeneous, i.e. $L_{i} \sim \operatorname{Exp}(\ell), a_{i} \equiv \bar{\varpi}$ and $K_{i} \equiv K \geq 0$. The expected default number can hardly capture the full picture of the risk, and we have to look at the entire distribution. We choose $K=\infty, 1 / 8,0$ and plot the estimated probability mass function (PMF) of the total default number within the period of $[0, t]$ in Figure 7 , and the corresponding quantiles are reported in Table 4. More specifically, Cases $K=\infty, 1 / 8,0$ correspond to the noncontagion (i.e. Case I), partial contagion and full contagion (i.e. Case II), respectively. We can clearly observe that, when $K$ decreases, the contagion would become more pronounced and the tail of losses becomes heavier. The system could be more susceptible to contagion risk when capital buffer $K$ is eroded, and contagion effects magnify the content of risk. As summarised by Eisenberg and Noe (2001, p.1310), bank defaults may be driven by losses from market and credit risk (i.e. fundamental default), and bank defaults may, however, also be initiated by contagion as a consequence of other bank failures in the system (i.e. contagious default). The two types of defaults, under our contagion model (i.e. the self-exciting jump sizes are not all equal to zeros), in fact, are mixed and interacting with each other. 

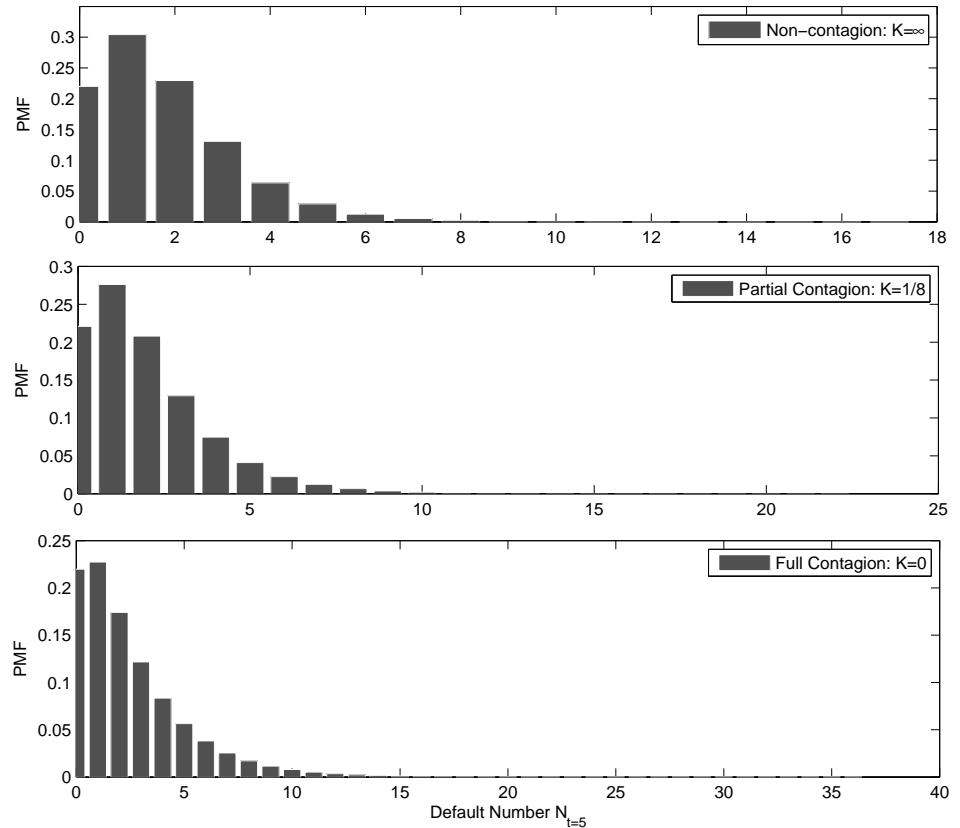

Figure 7: Probability mass function (PMF) of the default number $N_{t=5}$, estimated from $10^{6}$ replications based on the parameter setting $\left(\delta, \varrho ; a, b ; \ell, \bar{\varpi} ; \lambda_{0}\right)=(0.5,0.5 ; 0.5,2.0 ; 8.0,2.0 ; 0.5)$, with homogenous contagion thresholds $K=\infty, 1 / 8,0$, respectively; the associated quantiles are reported in Table 4

\subsection{A Model with Explosive Defaults}

Contagion or feedback effects could be even further reinforced due to highly leveraged positions (e.g. complicated credit derivatives), and the resulting system thereby becomes explosive, see discussions on the impacts of financial innovations in Corsi et al. (2016, p.1085). This scenario would be extremely severe, rare but possible, i.e. the entire system is not stable and near the boundary of crash. Mathematically, it corresponds to the non-stationary case when $\eta<0$ in our models. This may due to the "liquidity black holes" or "fire sales" of assets: it further depresses prices and leads to a sharp drop in liquidity and may also bring other institutions to fail in a self-reinforcing vicious spiral (?Krishnamurthy, 2010; Cont and Wagalath, 2013, 2016). All previous examples were conducted under the stationary condition $\eta>0$, and in fact our algorithms can also deal with non-stationary cases. In Figure 8, we offer three representative examples of $\eta=1 / 4,0,-1 / 2$ (or $\ell=8,4,2)$ for stationary, critical and explosive phases, respectively, and the associated quantiles are reported in Table 5. In particular, $\eta=0$ is the critical level of stability. The resulting loss distributions could present heavy tails, which might be very desirable for many regulators and practitioners. 

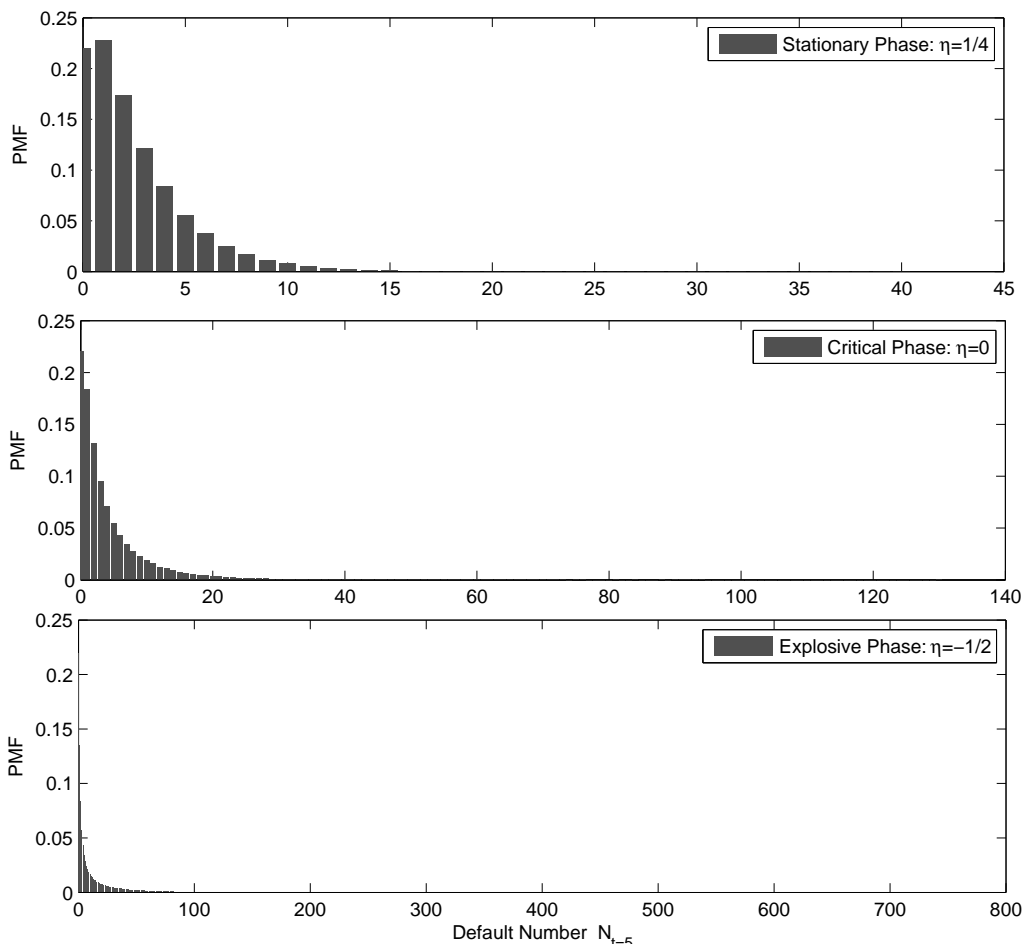

Figure 8: Probability mass function (PMF) of the default number $N_{t=5}$, estimated from $10^{6}$ replications based on the parameter setting $\left(\delta, \varrho ; a, b ; \bar{\varpi} ; \lambda_{0}\right)=(0.5,0.5 ; 0.5,2.0 ; 2.0 ; 0.5)$, with $\ell=8,4,2$, respectively; the associated quantiles are reported in Table 5

Table 5: Quantiles of the default number $N_{t=5}$, estimated from $10^{6}$ replications based on the parameter setting $\left(\delta, \varrho ; a, b ; \bar{\varpi} ; \lambda_{0}\right)=(0.5,0.5 ; 0.5,2.0 ; 2.0 ; 0.5)$, with $\ell=8,4,2$, respectively

\begin{tabular}{|c|c|c|c|c|c|c|c|c|}
\hline$C_{\ell} \quad$ Quantile & $5 \%$ & $25 \%$ & $50 \%$ & $75 \%$ & $95 \%$ & Mean & Min & Max \\
\hline 8 & 0 & 1 & 2 & 4 & 8 & 2.4969 & 0 & 40 \\
\hline 4 & 0 & 1 & 2 & 5 & 15 & 4.0628 & 0 & 130 \\
\hline 2 & 0 & 1 & 4 & 16 & 73 & 15.5662 & 0 & 743 \\
\hline
\end{tabular}




\subsection{Other Models}

In fact, our models and the associated algorithms could be further extended in several other directions. Due to the space limit, we briefly discuss as follows.

- A model with credit improvement: In this setup, we allow for the possibility of credit improvement or relief. For example, when a big loss occurs, a rescue plan may be released such as a "bailout" or a large cash injection into the system to ensure liquidity provision. This might significantly enhance the financial system in a relatively short term, and the intensity level may have an immediate decline instead of a climb, i.e.,

$$
\lambda_{T_{i}}=d_{i} \lambda_{T_{i}^{-}}
$$

where $d_{i}>0$ is a multiplier (which could be assumed to be a positive random variable). This model allows the intensity to jump in two sides, which can be simply generated by replacing (3.14) in the Step 4 of Algorithm 3.3 by (6.4).

- A model with structure breaks: A severe financial failure could make a large impact to the entire economic environment. For example, the collapse of the U.S. investment banking giant Lehman Brothers in Fall 2008 marked a clear tipping point of the entire financial market around the world. This would immediately act on the default intensity process and cause a structure break for the whole financial system. To model this pattern, we have to go beyond the original definition of the underlying intensity process (2.2), but our algorithms still can handle it easily, i.e. after each self-exciting jump, all parameters $\Theta$ afterward can be resetted to mimic a structure break. We can assign a new parameter set $\Theta_{i}$ immediately after the $i^{\text {th }}$ defaults. $\Theta_{i}$ could depend on the value of the size of the $i^{\text {th }}$ self-exciting jump $X_{i}$, or, even its whole history. So, the underlying intensity process (2.2) should be redefined locally based on the interarrival intensity (2.1) between two successive default times rather than globally throughout the positive real line $t \in \mathbb{R}^{+}$. Let us illustrate a simple example, say, there are two economic states after each self-exciting jump, one may correspond to a deteriorating economic environment and the other is to an improved one. We can use the parameter settings of $\Theta_{1}$ and $\Theta_{2}$ to model these two states respectively. We can choose one to be stable (i.e. stationary case $\eta>0$ ) and the other to be unstable (i.e. non-stationary case $\eta>0$ ). Then, the entire system could shift between locally stable and locally explosive phases. Analysis for contagion risk based on the stability of branching processes and allowing for a shift between two phases can also be found in Caccioli et al. (2014) and Corsi et al. (2016). 
- A model with multiple exogenous risk drivers: In practice, there may be multiple risk factors, such as sector-wide or market-wide events, commonly shared by all institutions. Multi-factor models then are required for modelling intensity processes, see e.g. Duffie and Gârleanu (2001), Das et al. (2007) and Longstaff and Rajan (2008). We could use a superposition of OU intensity processes driven by different gamma processes to capture the corresponding multiple risk factors ${ }^{13}$. Accordingly, our algorithms may be extendable to this version by using the superposition theory of point processes (Daley and Vere-Jones, 2003, Theorem 2.4.VI).

- A model with multilateral contagion: Contagion not only occurs within one market (or system, network) but could also spread across different markets. For example, when the loss contagion and investors' fears occur in the options market, it may also spread to the market of the underlying equity or futures on which the options are written. This type of contagion can be captured by adding mutually-exciting jumps. Similar as the multivariate Hawkes process, a multi-dimensional $\Gamma$-contagion process has to be developed to capture self-contagion effects for each individual, as well as the mutual contagion effects among them.

\section{Conclusion}

In this paper, we have introduced a new family of self-exciting jump processes whose intensities are driven by non-Gaussian OU processes, namely, Lévy-driven contagion processes. Backed by the very large family of Lévy subordinators, it indeed offers much richer choices beyond the classical Hawkes process for modelling the "contagion" of event arrivals in a continuous-time setup in finance, economics and many other fields. We have derived some important distributional properties of these new processes which lead to an exact simulation framework in general. In particular, we have developed exact simulation algorithms by decomposition approach for the gamma and tempered stable cases as typical examples. The algorithms are accurate and efficient which have been numerically verified and tested by extensive numerical experiments. We also provide applications to portfolio risk management, which again illustrate the efficiency, accuracy, applicability and flexibility of our algorithms. As a class of reduced-form models, it could be easily extended to pricing financial derivatives, particularly multiple-name credit products (e.g. collateralized debt obligations and mortgage-backed securities). It can be employed empirically when input data is available for parameter calibration. Furthermore, it could be widely applied to many other areas,

\footnotetext{
${ }^{13}$ Similarly, the superposition of OU stochastic volatility processes was proposed in Barndorff-Nielsen (2001) and Barndorff-Nielsen and Shephard (2001b, 2002).
} 
for example, to describe high-frequency trading data in market microstructure, claim arrivals for an insurance portfolio, or jump propagation, disclosure dynamics in financial markets (?). Their statistical inference and econometric analysis for this new framework, and further extensions to multidimensional point processes for modelling multilateral contagion, as well as further applications and empirical work for portfolio credit risk analysis, could be very interesting and meaningful topics for future research.

\section{Acknowledgments}

The authors would like to thank all reviewers for very helpful and constructive comments and suggestions. The corresponding author Hongbiao Zhao would like acknowledge the financial support from the National Natural Science Foundation of China (\#71401147) and the research funds provided by Shanghai University of Finance and Economics and Shanghai Institute of International Finance and Economics.

\section{Appendices}

\section{A Basic Distributional Properties}

In this section, we derive some basic distributional properties such as Laplace transforms and means to characterise this new family in general. The means will also be used for validating the associated simulation algorithms. First, let us provide the Laplace transform of intensity process as below.

Proposition A.1 (Laplace Transform of Intensity Process). Under the condition $\delta>\mu_{G}$, i.e. $\eta>0$, the Laplace transform of $\lambda_{t+s}$ conditional on $\lambda_{t}$ is given by

$$
\mathbb{E}\left[e^{-v \lambda_{t+s}} \mid \lambda_{t}\right]=\exp \left(-\mathcal{G}_{v}^{-1}(s) \lambda_{t}-\varrho \int_{\mathcal{G}_{v}^{-1}(s)}^{v} \frac{\Phi(u)}{\delta u+\hat{g}(u)-1} \mathrm{~d} u\right), \quad s>0
$$

where $\mathcal{G}_{v}^{-1}(\cdot)$ is the well-defined inverse function of

$$
\mathcal{G}_{v}(x):=\int_{x}^{v}[\delta u+\hat{g}(u)-1]^{-1} \mathrm{~d} u
$$

The Laplace transform of the asymptotic and stationary intensity process is given by

$$
\lim _{t \rightarrow \infty} \mathbb{E}\left[e^{-v \lambda_{t}}\right]=\exp \left(-\varrho \int_{0}^{v} \frac{\Phi(u)}{\delta u+\hat{g}(u)-1} \mathrm{~d} u\right)
$$


Proof. The infinitesimal generator of $\left(\lambda_{t}, N_{t}, t\right)$ acting on any function $f(\lambda, n, t)$ within its domain $\Omega(\mathcal{A})$ is given by

$$
\begin{aligned}
\mathcal{A} f(\lambda, n, t)= & \frac{\partial f}{\partial t}-\delta \lambda \frac{\partial f}{\partial \lambda}+\varrho\left\{\int_{0}^{\infty}[f(\lambda+z, n, t)-f(\lambda, n, t)] \nu(\mathrm{d} z)\right\} \\
+\lambda & {\left[\int_{0}^{\infty} f(\lambda+y, n+1, t) \mathrm{d} G(y)-f(\lambda, n, t)\right], }
\end{aligned}
$$

see more details on using infinitesimal generators in Davis (1984, 1993) and Duffie et al. (2003). Using the martingale approach similarly as Dassios and Zhao (2011, Theorem 3.2, p.819-821), it is easy to obtain (A.1). Note that, we have $\mathcal{G}_{v}^{-1}(t) \rightarrow 0$ when $t \rightarrow \infty$, then, the Laplace transform becomes independent of the time and the initial intensity, so we have (A.3).

Note that, the interarrival intensity process, i.e, the intensity process between two consecutive selfexciting jumps (excluding self-exciting jumps), is simply a Lévy-driven OU process. Hence, given the arrival times $\left\{T_{i}\right\}_{i=1,2, \ldots}$, the Laplace transform of $\lambda_{T_{i}+s}$ conditional on the intensity level $\lambda_{T_{i}}$ for any fixed time $s$ within the time period $\left(0, T_{i+1}-T_{i}\right)$ can be expressed more nicely as below, by setting $\hat{g}(u)=1$ in (A.1) to eliminate all self-exciting jumps.

Corollary A.1 (Laplace Transform of Interarrival Intensity Process). Given the $i^{\text {th }}$ and the $(i+1)^{\text {th }}$ arrival times $T_{i}$ and $T_{i+1}$ respectively, the Laplace transform of $\lambda_{T_{i}+\tau}$ conditional on $\lambda_{T_{i}}$ is given by

$$
\mathbb{E}\left[e^{-v \lambda_{T_{i}+\tau}} \mid \lambda_{T_{i}}\right]=\exp \left(-v e^{-\delta \tau} \lambda_{T_{i}}-\frac{\varrho}{\delta} \int_{w v}^{v} \frac{\Phi(u)}{u} \mathrm{~d} u\right), \quad \tau \in\left(0, T_{i+1}-T_{i}\right)
$$

The mean of point process is provided in Proposition 2.1 with the proof as below.

Proof. The mean of $N_{t+s}$ conditional on $N_{t}$ and $\lambda_{t}$ immediately follows by calculating

$$
\mathbb{E}\left[N_{t+s} \mid N_{t}, \lambda_{t}\right]=N_{t}+\mathbb{E}\left[\int_{s}^{t+s} \lambda_{u} \mathrm{~d} u \mid \lambda_{t}\right]=N_{t}+\int_{s}^{t+s} \mathbb{E}\left[\lambda_{u} \mid \lambda_{t}\right] \mathrm{d} u,
$$

where

$$
\mathbb{E}\left[\lambda_{t+s} \mid \lambda_{t}\right]= \begin{cases}\frac{\varrho \mu_{Z}}{\eta}+\left(\lambda_{t}-\frac{\varrho \mu_{Z}}{\eta}\right) e^{-\eta s}, & \eta \neq 0 \\ \lambda_{t}+\varrho \mu_{Z} s, & \eta=0\end{cases}
$$

which can be derived by differentiating the conditional Laplace transform (A.1). 


\section{B A/R Scheme for Exact Simulation of Random Variable $W$}

Algorithm B.1 (A/R Scheme for $W)$. The random variable $W$ with density

$$
f_{W}(u)=\frac{1}{C_{w}} \frac{\ln u}{\vartheta u-\frac{1}{\delta}} \boldsymbol{1}_{\left\{1, \frac{1}{w}\right\}}
$$

can be exactly simulated by the following $A / R$ procedure:

1. Generate a random variable

$$
E_{e}=w^{-\sqrt{U_{0}}}, \quad U_{0} \sim U[0,1]
$$

2. Generate a standard uniform random variable $U \sim U[0,1]$;

3. If

$$
U \leq \frac{b E_{e}}{\vartheta E_{e}-\frac{1}{\delta}}
$$

then, accept and set $W=E_{e}$; Otherwise, reject this candidate and go back to Step 1.

Proof. Note that,

$$
\vartheta u-\frac{1}{\delta}=b u+\frac{1}{\delta}(u-1) \geq b u, \quad u \in\left[1, \frac{1}{w}\right]
$$

then, we have

$$
f_{W}(u)=\frac{1}{C_{w}} \frac{\ln u}{\vartheta u-\frac{1}{\delta}} \leq \frac{1}{C_{w}} \frac{\ln u}{b u} .
$$

The density function of the envelop $E_{e}$ is

$$
f_{E_{e}}(u)=\frac{1}{E_{w}} \frac{\ln u}{u}, \quad E_{w}:=\frac{1}{2} \ln ^{2} w, \quad u \in\left[1, \frac{1}{w}\right],
$$

and the $\mathrm{CDF}$ is

$$
F_{E_{e}}(u)=\left(\frac{\ln u}{\ln w}\right)^{2}, \quad u \in\left[1, \frac{1}{w}\right]
$$

which has the analytic inverse

$$
F_{E_{e}}^{-1}(x)=w^{-\sqrt{x}}, \quad x \in[0,1] .
$$

Therefore, we have

$$
\frac{f_{W}(u)}{f_{E_{e}}(u)}=\frac{\frac{1}{C_{w}} \frac{\ln u}{\vartheta u-\frac{1}{\delta}}}{\frac{1}{E_{w}} \frac{\ln u}{u}} \leq \frac{\frac{1}{C_{w}} \frac{\ln u}{b u}}{\frac{1}{E_{w}} \frac{\ln u}{u}}=\frac{1}{b} \frac{E_{w}}{C_{w}}=\frac{\ln ^{2} w}{2 b C_{w}}:=\bar{c}_{w}
$$

and the acceptance condition for the A/R scheme is

$$
U \leq \frac{1}{\bar{c}_{w}} \frac{f_{W}\left(E_{e}\right)}{f_{E_{e}}\left(E_{e}\right)}=b \frac{E_{e}}{\vartheta E_{e}-\frac{1}{\delta}}
$$




\section{A/R Scheme for Exact Simulation of Random Variable $V$}

Algorithm C.1 (A/R Scheme for $V)$. The random variable $V$ with density (4.19) can be exactly simulated by the following $A / R$ procedure:

1. Generate a random variable

$$
E_{e}=\left\{\frac{1}{2}\left[\left(\alpha C_{w} U_{3}+2\right)+\sqrt{\left(\alpha C_{w} U_{3}+2\right)^{2}-4}\right]\right\}^{\frac{1}{\alpha}}, \quad U_{3} \sim U[0,1]
$$

where

$$
C_{w}=\frac{1}{\alpha}\left(w^{-\alpha}+w^{\alpha}-2\right)
$$

2. Generate a standard uniform random variable $U_{4} \sim U[0,1]$.

3. If

$$
U_{4} \leq \frac{\beta^{1-\alpha}}{E_{e}^{\alpha-1}-E_{e}^{-1-\alpha}} \frac{1-E_{e}^{-\alpha}}{\left(\kappa E_{e}-\frac{1}{\delta}\right)^{1-\alpha}},
$$

then, accept and set $V=E_{e}$; Otherwise, reject this candidate and go back to Step 1 .

Proof. The density of $V$ in (4.19) can be rewritten by

$$
f_{V}(u)=\frac{1}{D_{w}} \frac{1}{\kappa^{1-\alpha}} \frac{1-u^{-\alpha}}{\left(u-\frac{1}{\delta \kappa}\right)^{1-\alpha}}, \quad u \in\left[1, \frac{1}{w}\right]
$$

By introducing a constant $\xi$ such that

$$
\xi \geq \frac{u^{1-\alpha}}{\left(u-\frac{1}{\delta \kappa}\right)^{1-\alpha}}, \quad \forall u \in\left[1, \frac{1}{w}\right]
$$

we have

$$
f_{V}(u)<\frac{1}{D_{w}} \frac{\xi}{\kappa^{1-\alpha}}\left[u^{-(1-\alpha)}-u^{-(1+\alpha)}\right], \quad \forall u \in\left[1, \frac{1}{w}\right]
$$

Since the function $\frac{u^{1-\alpha}}{\left(u-\frac{1}{\delta \kappa}\right)^{1-\alpha}}$ is a strictly decreasing function of $u \in\left[1, \frac{1}{w}\right]$, i.e.

$$
\frac{\mathrm{d}}{\mathrm{d} u}\left[\frac{u^{1-\alpha}}{\left(u-\frac{1}{\delta \kappa}\right)^{1-\alpha}}\right]=(\alpha-1) \frac{1}{\delta \kappa} u^{-\alpha}\left(u-\frac{1}{\delta \kappa}\right)^{\alpha-2}<0
$$

we have $\xi \geq\left(\frac{\kappa}{\beta}\right)^{1-\alpha}$ for any $u \in\left[1, \frac{1}{w}\right]$, and then,

$$
\xi \geq \max _{1 \leq u \leq \frac{1}{w}}\left\{\frac{u^{1-\alpha}}{\left(u-\frac{1}{\delta \kappa}\right)^{1-\alpha}}\right\}=\left(\frac{\kappa}{\beta}\right)^{1-\alpha}
$$

We choose $E_{e}$ to be the envelop random variable with density

$$
g_{E_{e}}(u)=\frac{1}{C_{w}}\left[u^{-(1-\alpha)}-u^{-(1+\alpha)}\right], \quad u \in\left[1, \frac{1}{w}\right]
$$


Its CDF is

$$
F_{E_{e}}(u)=\frac{1}{\alpha C_{w}}\left(u^{-\alpha}+u^{\alpha}-2\right), \quad u \in\left[1, \frac{1}{w}\right]
$$

which has an explicit inverse function

$$
F_{E_{e}}^{-1}(x)=\left\{\frac{1}{2}\left[\left(\alpha C_{w} x+2\right)+\sqrt{\left(\alpha C_{w} x+2\right)^{2}-4}\right]\right\}^{\frac{1}{\alpha}}, \quad x \in[0,1]
$$

Hence, $E_{e}$ can be exactly simulated via the explicit inverse transform (C.1). Setting $\xi=\left(\frac{\kappa}{\beta}\right)^{1-\alpha}$, we have the acceptance rate (i.e. the expected number of candidates generated until one is accepted)

$$
\bar{c}_{w}=\frac{\xi}{\kappa^{1-\alpha}} \frac{C_{w}}{D_{w}}=\beta^{1-\alpha} \frac{C_{w}}{D_{w}} \geq \frac{f_{V}(u)}{g_{E_{e}}(u)} .
$$

We have also carried out some numerical tests for Algorithm C.1 and have found that it can achieve a high level of efficiency and accuracy. For example, it only takes about 7 seconds to generate $10^{6}$ replications with percentage error $0.1 \%$ for the parameter setting $(\delta, \varrho, \alpha, \beta, \theta)=(0.5,1,0.9,0.2,0.25)$.

\section{Backward Recursive (BR) Scheme for Stable Index $\alpha=1 / 4$}

Algorithm D.1 (Backward Recursive (BR) Scheme). For simulating one random variable of TS $\left(\alpha=\frac{1}{4}, \beta, \theta\right)$ :

1. Simulate an IG random variable

$$
S_{2} \sim \operatorname{IG}\left(2 \theta \beta^{-\frac{1}{4}} \Gamma\left(\frac{3}{4}\right), 8 \theta^{2} \Gamma^{2}\left(\frac{3}{4}\right)\right)
$$

2. Conditional on one realisation of $S_{2}$, simulate another $I G$ random variable

$$
S_{1} \mid S_{2} \sim I G\left(\frac{1}{2} \beta^{-\frac{1}{2}} S_{2}, \frac{1}{2} S_{2}^{2}\right)
$$

3. The resulting random variable $S_{1}$ is exactly equal in distribution to $\operatorname{TS}\left(\alpha=\frac{1}{4}, \beta, \theta\right)$.

Note that, IG random variables can be very efficiently simulated without $A / R$ mechanism using the classical algorithm developed by Michael et al. (1976). See the proof of this algorithm and other choices of the stable index $\alpha$ in Dassios et al. (2018).

\section{E Simple Stable Rejection (SSR) Scheme}

Algorithm E.1 (Simple Stable Rejection (SSR) Scheme). For simulating one random variable TS $\operatorname{TS}(\alpha, \beta, \theta)$ : 
1. Generate a stable random variable $S(\alpha, \theta)$ via

$$
S(\alpha, \theta) \stackrel{\mathcal{D}}{=}(-\theta \Gamma(-\alpha))^{\frac{1}{\alpha}} \frac{\sin \left(\alpha U_{s}+\frac{1}{2} \pi \alpha\right)}{\left(\cos \left(U_{s}\right)\right)^{\frac{1}{\alpha}}}\left[\frac{\cos \left((1-\alpha) U_{s}-\frac{1}{2} \pi \alpha\right)}{E_{s}}\right]^{\frac{1-\alpha}{\alpha}}
$$

where $U_{s} \sim U\left[-\frac{1}{2} \pi, \frac{1}{2} \pi\right], E_{s} \sim \operatorname{Exp}(1)$, and they are independent;

2. Generate a uniformly distributed random variable $U \sim U[0,1]$;

3. If $U \leq e^{-\beta S(\alpha, \theta)}$, then, accept and set $T S=S(\alpha, \theta)$; otherwise, reject and go back to Step 1 .

\section{References}

Aït-Sahalia, Y., Cacho-Diaz, J., and Laeven, R. J. (2015). Modeling financial contagion using mutually exciting jump processes. Journal of Financial Economics, 117(3):585-606.

Ait-Sahalia, Y. and Jacod, J. (2009). Estimating the degree of activity of jumps in high frequency data. Annals of Statistics, 37(5A):2202-2244.

Aït-Sahalia, Y. and Jacod, J. (2011). Testing whether jumps have finite or infinite activity. Annals of Statistics, 39(3):1689-1719.

Aït-Sahalia, Y. and Jacod, J. (2014). High-Frequency Financial Econometrics. Princeton University Press.

Aït-Sahalia, Y., Laeven, R. J., and Pelizzon, L. (2014). Mutual excitation in Eurozone sovereign CDS. Journal of Econometrics, 183(2):151-167.

Asmussen, S. and Glynn, P. W. (2007). Stochastic Simulation: Algorithms and Analysis. Springer.

Azizpour, S., Giesecke, K., and Schwenkler, G. (2018). Exploring the sources of default clustering. Journal of Financial Economics, 129(1):154-183.

Bacry, E., Delattre, S., Hoffmann, M., and Muzy, J.-F. (2013). Modelling microstructure noise with mutually exciting point processes. Quantitative Finance, 13(1):65-77.

Barndorff-Nielsen, O. and Shephard, N. (2001a). Modelling by Lévy processess for financial econometrics. In Barndorff-Nielsen, O., Resnick, S., and Mikosch, T., editors, Lévy Processes, pages 283-318. Birkhäuser, Boston.

Barndorff-Nielsen, O. E. (1997). Normal inverse Gaussian distributions and stochastic volatility modelling. Scandinavian Journal of Statistics, 24(1):1-13.

Barndorff-Nielsen, O. E. (1998). Processes of normal inverse Gaussian type. Finance and Stochastics, 2(1):41-68.

Barndorff-Nielsen, O. E. (2001). Superposition of Ornstein-Uhlenbeck type processes. Theory of Probability \& Its Applications, 45(2):175-194. 
Barndorff-Nielsen, O. E., Jensen, J. L., and Sørensen, M. (1998). Some stationary processes in discrete and continuous time. Advances in Applied Probability, 30(4):989-1007.

Barndorff-Nielsen, O. E. and Shephard, N. (2001b). Non-Gaussian Ornstein-Uhlenbeck-based models and some of their uses in financial economics. Journal of the Royal Statistical Society: Series B (Statistical Methodology), 63(2):167-241.

Barndorff-Nielsen, O. E. and Shephard, N. (2002). Econometric analysis of realized volatility and its use in estimating stochastic volatility models. Journal of the Royal Statistical Society: Series B (Statistical Methodology), 64(2):253-280.

Barndorff-Nielsen, O. E. and Shephard, N. (2003a). Integrated OU processes and non-Gaussian OU-based stochastic volatility models. Scandinavian Journal of Statistics, 30(2):277-295.

Barndorff-Nielsen, O. E. and Shephard, N. (2003b). Realized power variation and stochastic volatility models. Bernoulli, 9(2):243-265.

Bowsher, C. G. (2007). Modelling security market events in continuous time: Intensity based, multivariate point process models. Journal of Econometrics, 141(2):876-912.

Brémaud, P. and Massoulié, L. (1996). Stability of nonlinear Hawkes processes. The Annals of Probability, 24(3):1563-1588.

Brémaud, P. and Massoulié, L. (2002). Power spectra of general shot noises and Hawkes point processes with a random excitation. Advances in Applied Probability, 34(1):205-222.

Brix, A. (1999). Generalized gamma measures and shot-noise Cox processes. Advances in Applied Probability, 31(4):929-953.

Broadie, M. and Kaya, Ö. (2006). Exact simulation of stochastic volatility and other affine jump diffusion processes. Operations Research, 54(2):217-231.

Brunnermeier, M. K. (2009). Deciphering the liquidity and credit crunch 2007-2008. The Journal of Economic Perspectives, 23(1):77-100.

Brunnermeier, M. K. and Pedersen, L. H. (2009). Market liquidity and funding liquidity. Review of Financial Studies, 22(6):2201-2238.

Caccioli, F., Shrestha, M., Moore, C., and Farmer, J. D. (2014). Stability analysis of financial contagion due to overlapping portfolios. Journal of Banking \& Finance, 46:233-245.

Cai, N., Song, Y., and Chen, N. (2017). Exact simulation of the SABR model. Operations Research, 65(4):931-951.

Carr, P., Geman, H., Madan, D. B., and Yor, M. (2003). Stochastic volatility for Lévy processes. Mathematical Finance, 13(3):345-382. 
Centanni, S. and Minozzo, M. (2006). A Monte Carlo approach to filtering for a class of marked doubly stochastic Poisson processes. Journal of the American Statistical Association, 101(476):1582-1597.

Chen, N. and Huang, Z. (2013). Localization and exact simulation of Brownian motion-driven stochastic differential equations. Mathematics of Operations Research, 38(3):591-616.

Chen, Z., Feng, L., and Lin, X. (2012). Simulating Lévy processes from their characteristic functions and financial applications. ACM Transactions on Modeling and Computer Simulation, 22(3):14:1-14:26.

Chhikara, R. and Folks, L. (1989). The Inverse Gaussian Distribution: Theory, Methodology, and Applications. Marcel Dekker, New York.

Cont, R. (2001). Empirical properties of asset returns: stylized facts and statistical issues. Quantitative Finance, 1(2):223-236.

Cont, R. and Tankov, P. (2004). Financial Modelling with Jump Processes. CRC Press.

Cont, R. and Wagalath, L. (2013). Running for the exit: distressed selling and endogenous correlation in financial markets. Mathematical Finance, 23(4):718-741.

Cont, R. and Wagalath, L. (2016). Fire sales forensics: measuring endogenous risk. Mathematical Finance, 26(4):835-866.

Corsi, F., Marmi, S., and Lillo, F. (2016). When micro prudence increases macro risk: The destabilizing effects of financial innovation, leverage, and diversification. Operations Research, 64(5):1073-1088.

Cox, D. (1972). Regression models and life-tables. Journal of the Royal Statistical Society. Series B (Methodological), 34(2):187-220.

Cox, D. R. (1955). Some statistical methods connected with series of events. Journal of the Royal Statistical Society. Series B (Methodological), 17(2):129-164.

Crane, R. and Sornette, D. (2008). Robust dynamic classes revealed by measuring the response function of a social system. Proceedings of the National Academy of Sciences, 105(41):15649-15653.

CreditRisk $^{+}$(1997). CreditRisk ${ }^{+}$: A Credit Risk Management Framework. Credit Suisse First Boston International, New York.

Daley, D. J. and Vere-Jones, D. (2003). An Introduction to the Theory of Point Processes: Volume I: Elementary Theory and Methods. Springer, New York.

Das, S. R., Duffie, D., Kapadia, N., and Saita, L. (2007). Common failings: How corporate defaults are correlated. Journal of Finance, 62(1):93-117.

Dassios, A. and Embrechts, P. (1989). Martingales and insurance risk. Stochastic Models, 5(2):181-217.

Dassios, A., Qu, Y., and Zhao, H. (2018). Exact simulation for a class of tempered stable and related distributions. ACM Transactions on Modeling and Computer Simulation, 28(3):20:1-20:21. 
Dassios, A. and Zhao, H. (2011). A dynamic contagion process. Advances in Applied Probability, 43(3):814-846.

Dassios, A. and Zhao, H. (2013). Exact simulation of Hawkes process with exponentially decaying intensity. Electronic Communications in Probability, 18(62):1-13.

Dassios, A. and Zhao, H. (2017a). Efficient simulation of clustering jumps with CIR intensity. Operations Research, 65(6):1494-1515.

Dassios, A. and Zhao, H. (2017b). A generalised contagion process with an application to credit risk. International Journal of Theoretical and Applied Finance, 20(1):1-33.

Davis, M. H. (1984). Piecewise-deterministic Markov processes: A general class of non-diffusion stochastic models. Journal of the Royal Statistical Society. Series B (Methodological), 46(3):353-388.

Davis, M. H. (1993). Markov Models and Optimization. Chapman \& Hall/CRC, London.

Devroye, L. (2009). Random variate generation for exponentially and polynomially tilted stable distributions. ACM Transactions on Modeling and Computer Simulation, 19(4):1-20.

Duffie, D., Eckner, A., Horel, G., and Saita, L. (2009). Frailty correlated default. Journal of Finance, 64(5):2089-2123.

Duffie, D., Filipovic, D., and Schachermayer, W. (2003). Affine processes and applications in finance. Annals of Applied Probability, 13(3):984-1053.

Duffie, D. and Gârleanu, N. (2001). Risk and valuation of Collateralized Debt Obligations. Financial Analysts Journal, 57(1):41-59.

Eberlein, E., Madan, D., Pistorius, M., and Yor, M. (2013). A simple stochastic rate model for rate equity hybrid products. Applied Mathematical Finance, 20(5):461-488.

Eisenberg, L. and Noe, T. H. (2001). Systemic risk in financial systems. Management Science, 47(2):236249.

Elsinger, H., Lehar, A., and Summer, M. (2006). Risk assessment for banking systems. Management Science, 52(9):1301-1314.

Embrechts, P., Liniger, T., and Lin, L. (2011). Multivariate Hawkes processes: an application to financial data. Journal of Applied Probability, 48A:367-378.

Errais, E., Giesecke, K., and Goldberg, L. R. (2010). Affine point processes and portfolio credit risk. SIAM Journal on Financial Mathematics, 1(1):642-665.

Gençay, R., Dacorogna, M., Muller, U. A., Pictet, O., and Olsen, R. (2001). An Introduction to Highfrequency Finance. Academic Press. 
Giesecke, K., Kakavand, H., and Mousavi, M. (2011a). Exact simulation of point processes with stochastic intensities. Operations Research, 59(5):1233-1245.

Giesecke, K., Longstaff, F. A., Schaefer, S., and Strebulaev, I. (2011b). Corporate bond default risk: A 150-year perspective. Journal of Financial Economics, 102(2):233-250.

Glasserman, P. (2003). Monte Carlo Methods in Financial Engineering. Springer.

Glasserman, P. and Liu, Z. (2010). Sensitivity estimates from characteristic functions. Operations Research, 58(6):1611-1623.

Gordy, M. B. (2000). A comparative anatomy of credit risk models. Journal of Banking \& Finance, 24(1):119-149.

Gordy, M. B. (2003). A risk-factor model foundation for ratings-based bank capital rules. Journal of Financial Intermediation, 12(3):199-232.

Hainaut, D. and Devolder, P. (2008). Mortality modelling with Lévy processes. Insurance: Mathematics and Economics, 42(1):409-418.

Hawkes, A. G. (1971a). Point spectra of some mutually exciting point processes. Journal of the Royal Statistical Society. Series B (Methodological), 33(3):438-443.

Hawkes, A. G. (1971b). Spectra of some self-exciting and mutually exciting point processes. Biometrika, 58(1):83-90.

Hawkes, A. G. and Oakes, D. (1974). A cluster process representation of a self-exciting process. Journal of Applied Probability, 11:493-503.

Hofert, M. (2011). Sampling exponentially tilted stable distributions. ACM Transactions on Modeling and Computer Simulation, 22(1):1-11.

Kang, C., Kang, W., and Lee, J. M. (2017). Exact simulation of the wishart multidimensional stochastic volatility model. Operations Research, 65(5):1190-1206.

Krishnamurthy, A. (2010). How debt markets have malfunctioned in the crisis. The Journal of Economic Perspectives, 24(1):3-28.

Kyprianou, A. (2006). Introductory Lectures on Fluctuations of Lévy Processes with Applications. Springer.

Large, J. (2007). Measuring the resiliency of an electronic limit order book. Journal of Financial Markets, $10(1): 1-25$.

Lee, S. S. and Hannig, J. (2010). Detecting jumps from Lévy jump diffusion processes. Journal of Financial Economics, 96(2):271-290.

Lewis, P. A. and Shedler, G. S. (1979). Simulation of nonhomogeneous Poisson processes by thinning. Naval Research Logistics Quarterly, 26(3):403-413. 
Li, H., Wells, M. T., and Cindy, L. Y. (2008). A Bayesian analysis of return dynamics with Lévy jumps. Review of Financial Studies, 21(5):2345-2378.

Li, L. and Linetsky, V. (2014). Time-changed Ornstein-Uhlenbeck processes and their applications in commodity derivative models. Mathematical Finance, 24(2):289-330.

Longstaff, F. A. and Rajan, A. (2008). An empirical analysis of the pricing of collateralized debt obligations. Journal of Finance, 63(2):529-563.

Madan, D., Carr, P., and Chang, E. (1998). The variance gamma process and option pricing. European Finance Review, 2(1):79-105.

Madan, D. B. and Seneta, E. (1990). The variance gamma (V.G.) model for share market returns. Journal of Business, 63(4):511-524.

Michael, J. R., Schucany, W. R., and Haas, R. W. (1976). Generating random variates using transformations with multiple roots. The American Statistician, 30(2):88-90.

Nicolato, E. and Venardos, E. (2003). Option pricing in stochastic volatility models of the OrnsteinUhlenbeck type. Mathematical Finance, 13(4):445-466.

Poterba, J. M. and Summers, L. H. (1988). Mean reversion in stock prices: Evidence and implications. Journal of Financial Economics, 22(1):27-59.

Rosiński, J. (2001). Series representations of Lévy processes from the perspective of point processes. In Barndorff-Nielsen, O., Resnick, S., and Mikosch, T., editors, Lévy Processes, pages 401-415. Birkhäuser, Boston.

Rosiński, J. (2007). Tempering stable processes. Stochastic Processes and their Applications, 117(6):677707.

Rydberg, T. H. and Shephard, N. (2000). A modelling framework for the prices and times made on the NYSE. In Fitzgerald, W., Smith, R., Walden, A., and Young, P., editors, Nonlinear and Nonstationary Signal Processing, Issac Newton Institute Series. Cambridge University Press.

Sato, K.-i. (1999). Lévy Processes and Infinitely Divisible Distributions. Cambridge University Press.

Schoutens, W. and Cariboni, J. (2010). Lévy Processes in Credit Risk. John Wiley \& Sons. 